\title{
When are bacteria really gazelles? Comparing patchy ecologies with dimensionless numbers
}

Samuel S. Urmy*1 (urmy@mbari.org). Monterey Bay Aquarium Research Institute, 7700 Sandholdt Rd., Moss Landing, CA 95039, USA.

Alli N. Cramer*2 (ancramer@ucsc.edu). School of the Environment, Washington State University, Pullman, WA 99163, USA

Tanya L. Rogers (tanya.rogers@noaa.gov). NOAA Southwest Fisheries Science Center, 110 McAllister Way, Santa Cruz, CA 95060, USA

Jenna Sullivan-Stack (jenna.sullivan@oregonstate.edu). Department of Integrative Biology, Oregon State University, 3029 Cordley Hall, Corvallis, OR 97331, USA

Marian Schmidt (marschmi@umich.edu). Department of Integrative Biology, University of Texas at Austin, Texas 78712, USA

Simon D. Stewart (Simon.Stewart@cawthron.org.nz). Cawthron Institute, 98 Halifax St East, Nelson 7010, New Zealand

Celia C. Symons (csymons@uci.edu). University of California, Irvine, Department of Ecology and Evolutionary Biology, 469 Steinhaus Hall, Irvine, CA 92697, USA

* Authors contributed equally to the manuscript

${ }^{1}$ Current address: NOAA Alaska Fisheries Science Center, 7600 Sand Point Way NE, Seattle, WA 98115, USA

${ }^{2}$ Current address: Institute of Marine Science at University of California Santa Cruz \& NOAA Southwest Fisheries Science Center, 110 McAllister Way, Santa Cruz, CA 95060, USA

Data accessibility: All data and code to reproduce the analyses are available at https://github.com/allicramer/EcologicalPatchiness. 


\section{Abstract}

From micro to planetary scales, spatial heterogeneity-patchiness-is ubiquitous in ecological systems, defining the environments in which organisms move and interact. While this fact has been recognized for decades, most large-scale ecosystem models still use spatially averaged "mean fields" to represent natural populations, while fine-scale, spatially explicit models are mostly restricted to particular organisms or systems. In a conceptual paper, Grünbaum (2012, Interface Focus 2: 150-155) introduced a heuristic framework, based on three dimensionless ratios quantifying movement, reproduction, and resource consumption, to characterize patchy ecological interactions and identify when mean-field assumptions are justifiable. In this paper, we calculated Grünbaum's dimensionless numbers for 33 real interactions between consumers and their resource patches in terrestrial, aquatic, and aerial environments. Consumers ranged in size from bacteria to blue whales, and patches lasted from minutes to millennia, spanning spatial scales of $\mathrm{mm}$ to hundreds of $\mathrm{km}$. We found that none of the interactions could be accurately represented by a purely mean-field model, though 26 of them (79\%) could be partially simplified by averaging out movement, reproductive, or consumption dynamics. Clustering consumer-resource pairs by their non-dimensional ratios revealed several unexpected dynamic similarities between disparate interactions. For example, bacterial Pseudoalteromonas exploit nutrient plumes in a similar manner to Mongolian gazelles grazing on ephemeral patches of steppe vegetation. Our findings suggest that dimensional analysis is a valuable tool for characterizing ecological patchiness, and can link the dynamics of widely different systems into a single quantitative framework.

Keywords: Comparative ecology, Dimensional analysis, Interactions, Model systems, Patchiness, Scale 


\section{Introduction}

Most ecosystems are patchy across a range of spatial and temporal scales, and, consequently, most ecological interactions occur in patchy environments (Hutchinson 1961; Wiens 1976; Levin 1994; Lawton 1999). In the ocean, predators search for shifting schools of fish (Weimerskirch et al. 2005; Benoit-Bird et al. 2013). In the forest, grazers move from plant to plant or stand to stand (de Knegt et al. 2007). And at the micro-scale, bacteria search for organic particles and plumes of dissolved nutrients (Stocker 2012; Hellweger 2018; Yawata et al. 2020). The uneven distribution of resources means that consumers (i.e., predators, herbivores, or detritivores) often experience resources far from their mean density. Patchiness and heterogeneous dynamics have been recognized as critical issues in ecology for decades (Durrett \& Levin 1994; Codling \& Dumbrell 2012) and form the basis of many ecological theories (Chesson 2000; Davis et al. 2005). However, ecologists still struggle to incorporate them into our mental, mathematical, and management models.

Two main challenges have prevented the full integration of patchiness into ecology and management. First, patches are by definition localized in space and time, so incorporating them requires temporally and spatially explicit models. Spatially explicit models are more theoretically complex, computationally expensive, and difficult to use than non-spatial models (Morozov \& Poggiale 2012; DeAngelis \& Yurek 2017). Consequently, most researchers still rely on "mean-field" models, assuming that spatially variable quantities can be represented by a single average value (Codling \& Dumbrell 2012). Despite their simplicity and wide use, mean-field models are often inadequate descriptions of real ecological dynamics (Priyadarshi et al. 2019). They are sensitive to functional responses at low population densities, often require arbitrary assumptions of ecological thresholds, and can erroneously predict competitive exclusions or extinctions in environments where real populations persist (Hutchinson 1961; Murray 1989; Grünbaum 2012). While mean-field approximations may be appropriate in some situations, they must be justified based on specific hypotheses and scales of ecological processes.

The second challenge arises because ecological patches occur across a vast range of physical scales. Patchiness is present in almost all systems at some scale, and many statistics and metrics exist to quantify it (Fortin 1999). However, it is less clear how to determine which patches, at which scales, are relevant for which organisms. Moreover, when a patchy process or interaction has been described for one species or system, it is often unclear which, if any, of its conclusions apply more generally. Such studies can remain isolated in their (sub)discipline's literature as apparent special cases, with no way to identify similar dynamics in other taxa or environments. 
Box 1 Dimensional analysis and dynamic similarity

$$
\mathrm{Re}=\frac{\text { Inertial Force }}{\text { Viscous Force }}
$$
Turbulent Flow
Re large,

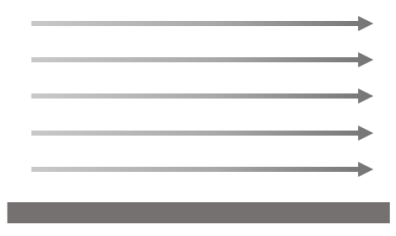

Re small, Laminar Flow

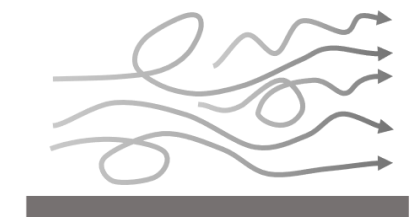

Dimensional analysis is a technique for simplifying mathematical representations of physical problems. The key to this method is to reduce the number of parameters required to describe a system by combining the original variables into a smaller number of dimensionless ratios-i.e., ratios where all the physical units cancel out, leaving only a pure number expressing the relative importance of its components. Despite widespread use in the physical sciences, dimensional analysis is still relatively rare in ecology (a notable exception being the relationship between growth, $K$, and mortality $M$, (Beverton \& Holt 1959; Charnov 1990; Charnov et al. 1991)).

Dimensional analysis can provide quantitative insight by identifying the importance of different variables. It can also provide qualitative insights by identifying fundamental properties of systems with similar dynamics. A well-known physical example is the Reynolds number $(\mathrm{Re})$, which gives the ratio of inertial to viscous forces within a fluid. Quantitatively, if $\mathrm{Re} \gg>1$ a fluid dynamicist can safely drop all frictional terms from their equations, even though some friction is, of course, always present. Qualitatively, flows with Re greater than about $5 \times 10^{5}$ are likely to be turbulent, and thus fundamentally different from the laminar flows prevalent at lower $\mathrm{Re}$. Since $\mathrm{Re}$ is unitless, this pattern holds true regardless of the particular fluid and physical scale. Thus, $R e$ is as useful for describing the biomechanics of plankton as it is for designing airplanes.

Systems are dynamically similar when the relative importance of their attributes are the same - i.e., the ratios between variables are the same. Dynamically similar systems can serve as models for one another, even if their absolute scales differ widely.

One approach capable of addressing both of these challenges is dimensional analysis, as it facilitates comparisons between processes, regardless of scale (Stephens \& Dunbar 1993; Horne \& Schneider 1994, Box 1). In a theoretical paper, Grünbaum (2012) proposed a dimensional analysis of interactions between consumers and their resource patches. In this framework, the resource landscape is simplified as a collection of discrete patches which are separated by a characteristic distance, persist for a characteristic time span, and contain a characteristic density of resources. Consumers are distinguished by their typical movement rates, generation times, and consumption rates (Figure 1). From these parameters, three dimensionless ratios can be calculated: the Frost, Strathmann, and Lessard numbers (Fr, Str, and Le, respectively; Table 1). These ratios compare the duration of an undisturbed patch to the typical time required for consumers to locate a patch (Fr), to reproduce (Str), and to consume a patch (Le). The three ratios summarize the importance of movement, reproduction, and resource depletion to the consumer-resource interaction, independent of the absolute scales and rates involved. 
Table 1 - Dimensionless ratios from Grünbaum (2012). In each ratio, $T$ represents the typical patch duration, $T_{\text {search }}$ is the time required for a consumer to locate a patch, $T_{\text {repro. }}$ is the consumer's generation time, and $T_{\text {cons. }}$ is the time required for consumers to deplete a patch. All of these durations are "characteristic time scales," accurate to an order of magnitude but not necessarily more so.

\begin{tabular}{llll}
\hline Dimensionless ratio & Description & Ratio $>1$ & Ratio $<<1$ \\
\hline $\begin{array}{l}\text { Frost number } \\
F r=T / T_{\text {search }}\end{array}$ & $\begin{array}{l}\text { The importance of forager } \\
\text { movement to resource patch } \\
\text { occupancy }\end{array}$ & $\begin{array}{l}\text { Consumers find } \\
\text { patches easily } \\
\text { and spend most } \\
\text { time inside them }\end{array}$ & $\begin{array}{l}\text { Consumers spend } \\
\text { most time } \\
\text { searching for } \\
\text { patches }\end{array}$ \\
$\begin{array}{llll}\text { Strathmann number } \\
\text { Str }=T / T_{\text {repro. }}\end{array}$ & $\begin{array}{l}\text { The importance of forager } \\
\text { reproduction to resource } \\
\text { patch occupancy }\end{array}$ & $\begin{array}{l}\text { Consumer } \\
\text { populations can } \\
\text { grow inside }\end{array}$ & $\begin{array}{l}\text { Consumers must } \\
\text { visit many } \\
\text { patches }\end{array}$ \\
$\begin{array}{llll}\text { Lessard number } \\
\text { Le }=T / T_{\text {cons. }}\end{array}$ & $\begin{array}{l}\text { The importance of forager } \\
\text { consumption to resource } \\
\text { patch persistence }\end{array}$ & $\begin{array}{l}\text { Consumers } \\
\text { actively deplete } \\
\text { patch resources }\end{array}$ & $\begin{array}{l}\text { Patch resource } \\
\text { densities not } \\
\text { affected by } \\
\text { consumption }\end{array}$ \\
\hline
\end{tabular}

The most practical application of these ratios is to determine which consumer-resource interactions can be approximated using a mean-field model. If Fr, Str, and Le are "small" (i.e., $<<$ 1 ), then consumer movement, reproduction, and consumption operate on time scales much longer than those of individual resource patches. Consumers encounter patches randomly and integrate over many patches in time, justifying the mean-field assumption. On the other hand, if any of the ratios are not "small," then the interaction is "functionally patchy," and must be treated as such. For instance, if $\mathrm{Fr}>>1$, consumers will spend most of their time inside patches, experiencing local resource densities well above the average value. In circumstances like these, mean-field assumptions underestimate the resources available to the consumers (Lasker 1975; Benoit-Bird et al. 2013). Grünbaum (2012) also proposed several conjectures for patchy interactions. For instance, consumer populations should only persist if they can locate patches easily, reproduce within patches, or both. This leads to the prediction that all interactions should have at least one of Fr or Str >1. Relatedly, if either movement or reproduction is difficult or costly for a consumer species, evolution should select for movement or reproductive rates that are "just fast enough" to raise Fr or Str above the critical value of $\approx 1$.

Despite their conceptual power and potential utility, to our knowledge these dimensionless ratios have never been empirically calculated, which was the objective of this study. We conducted a literature review and meta-analysis of patchy consumer-resource interactions across a wide range of taxa, scales, and ecosystems. We obtained values for consumer rates and the spatial and temporal scales of their resource patches, and used these to calculate Frost, Strathmann, and Lessard numbers for each interaction. Using these ratios, we determined which interactions 
were functionally patchy and which could be approximated using mean-field assumptions, and we evaluated several of Grünbaum's conjectures. Finally, we identified consumer-resource interactions that were dynamically similar — that is, close together in Fr-Str-Le space. This approach identified commonalities which might otherwise be obscured by differences in taxon, environment, or spatiotemporal scale, shifting the focus from individual species towards generalized interactions between consumers, resources, and their environments. The ultimate goal of such an analysis is to enable more parsimonious models and a deeper, more general understanding of patch dynamics in ecology.

(b)

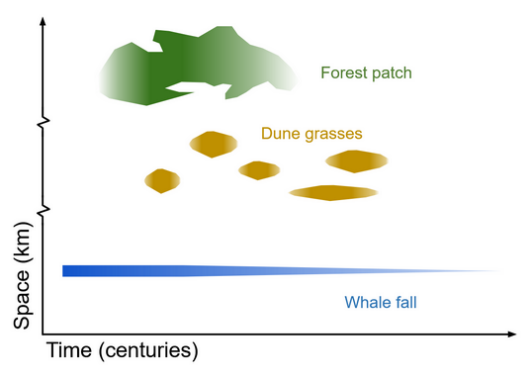

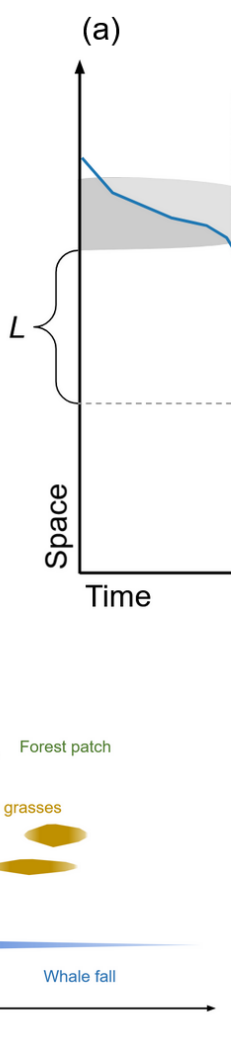
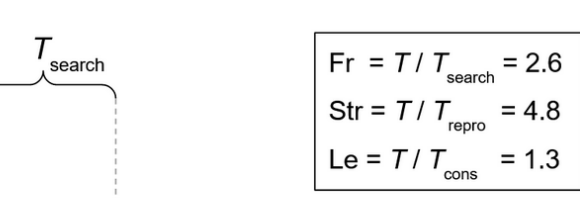

$$
\begin{aligned}
& \mathrm{Fr}=T / T_{\text {searc }}=2.6 \\
& \mathrm{Str}=T / T_{\text {repro }}=4.8 \\
& \mathrm{Le}=T / T_{\text {cons }}=1.3
\end{aligned}
$$
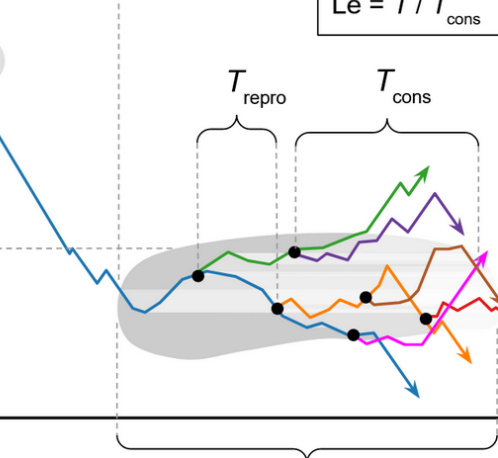

(c)

$T$

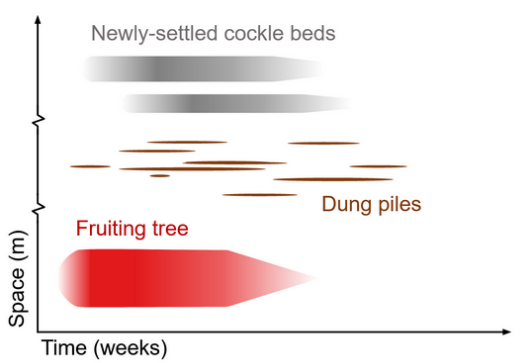

(d)

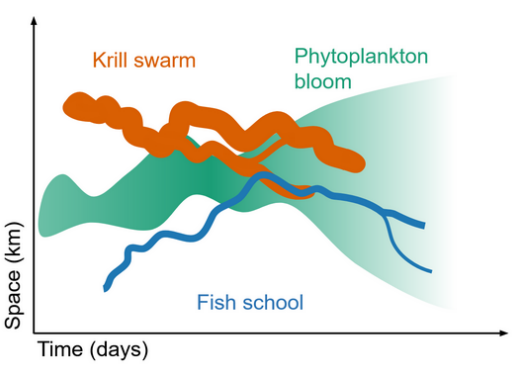

Figure 1. Conceptual space-time depictions of patchy consumer-resource interactions. (a) Hypothetical interaction between consumers (solid colored lines) feeding on resource patches (grey regions). As time (horizontal axis) progresses, consumers move on a simplified one-dimensional spatial domain (vertical axis), seeking resource patches. When they enter a patch, they begin to deplete it, indicated by lightened shading to the right of consumer trajectories (i.e., after their passage). Black dots represent reproduction events, and hence the birth of new consumers. Brackets show the characteristic duration of patches $(T)$ and the distance between them $(L)$, as well as the time scales for consumers to locate a patch ( $\left.T_{\text {search }}\right)$, reproduce ( $\left.T_{\text {repro }}\right)$, and consume a patch ( $T_{\text {cons }}$, assuming they are at a "typical" density within it). The box in the upper right gives the ratios of the interaction's time scales as its dimensionless Frost (Fr), Strathmann (Str), and Lessard (Le) numbers. Panels (b), (c), and (d) depict the different physical scales of various resource patches. (b) Forest stands, dune grass patches, and whale falls persist for tens or hundreds of years and are spaced at kilometer scales. (c) Trees bear ripe fruit, dung piles remain favorable for dung beetles, and newly settled cockles are small enough for crabs to eat for weeks, with spatial scales of meters. (d) Phytoplankton blooms persist for days to weeks, while zooplankton and fish aggregations last from hours to days, with spatial scales of km. 


\section{Methods}

We took a meta-study approach to calculate values of Grünbaum's dimensionless ratios for consumers feeding on patchy resources. For this analysis, a "patch" was defined as a spatially and temporally bounded region, within which a resource is dense enough for profitable exploitation by a consumer (Charnov 1976; Wiens 1976, Figure 2). Some researchers have used "patchiness" as a loose synonym for "heterogeneity," including spatial gradients (e.g., (Arditi \& Dacorogna 1988), but we required patches to contain a local maximum of the resource's density. We restricted our analysis to trophic interactions, where exploitation of the resource involved energy transfer to the consumer. For example, a hydrothermal vent is a resource patch for chemosynthetic organisms, because they use the vent fluids as a source of chemical energy. In contrast, a lake is not a resource patch for a fish-rather, it is a habitat.

To calculate values for Fr, Str, and Le, we searched the literature for data on specific consumer-resource pairs. Pairs were selected to reflect a diversity of systems, based on author expertise and availability of necessary data. Literature searches were performed using Google scholar and Web of Science for the parameters of interest (Table 2). Search terms included "speed", "turning interval", "movement", "growth rate", "reproduction", "consumption rate", "foraging", and "patch". We took values directly from published sources when possible, though some values were estimated based on published ranges or the interpretation of published figures. In the rare cases where data were not available, parameters were estimated either via the authors' expertise or consultation with experts. All raw values used for the analysis, with their sources, are tabulated in Appendix S1. While our selection of consumer-resource interactions cannot be considered random or unbiased, we made conscious efforts to represent marine, freshwater, and terrestrial environments; microbial, invertebrate, and vertebrate taxa employing carnivorous, herbivorous, and detrital feeding strategies; and patches which were stationary, passively moving, and actively moving.

These "characteristic" rates, scales, and densities for consumers and resource patches were necessarily broad-brush descriptions; selecting a single value based on the available literature was often challenging and somewhat arbitrary. Fortunately, for their intended use as descriptive heuristics, the dimensionless numbers did not require precision greater than 1-2 significant digits. With better data and domain knowledge it would be possible to calculate them more precisely, but was beyond the scope of this paper. In practice, the dimensionless ratios varied across many orders of magnitude (see Results and Discussion), so greater precision would not have qualitatively changed the results.

To allow for comparisons, we converted all rates and quantities to base SI units (seconds, meters, and grams) and checked for dimensional consistency. Values for Fr, Str, and Le were then calculated for each consumer-resource pair. We calculated two Frost numbers for each consumer: one based on an assumption of diffusive (i.e., random walk) movement, and one on 
directed-line (i.e., ballistic) movement. These two movement types are unrealistically simple, but define reasonable bounds for the range of more complex movement types used by real organisms.

To determine when systems are functionally patchy and to visualize the potential impact of that patchiness on consumer-resource dynamics, we developed four-quadrant diagrams similar to those in Grünbaum (2012). These diagrams provide a graphical assessment of patch dynamics and the importance of consumer movement (Fr), reproduction (Str), and patch consumption rate (Le) to trophic interactions. These three ratios can be visualized as a point in three dimensions; for readability, we display the ratios on logarithmic axes, and examine this 3-D point cloud projected on only two axes at a time. These figures also let us compare our results with predictions from Grünbaum (2012). We also calculated the Pearson product-moment correlation between each pair of dimensionless numbers.

Table 2. Parameters and rates obtained from the literature to develop data-derived Frost, Strathmann, and Lessard numbers. The physical dimensions of each quantity are denoted as $\mathbf{T}$ (time), $\mathbf{L}$ (length), $\mathbf{N}$ (number of individuals), and $\mathbf{M}$ (biomass), with the subscripts " $\mathrm{R}$ " and "C" indicating "resource" or "consumer." (Adapted from Table 1 in Grünbaum 2012.)

\begin{tabular}{|c|c|c|c|}
\hline & Parameter & Description & Dimension \\
\hline \multirow{4}{*}{$\begin{array}{l}\text { Consumer } \\
\text { level }\end{array}$} & $s$ & consumer speed & $\mathbf{L} \mathbf{T}^{-1}$ \\
\hline & $\tau$ & consumer turning interval & $\mathrm{T}$ \\
\hline & $\sigma$ & consumer generation time & $\mathrm{T}$ \\
\hline & $\eta$ & consumer-specific consumption rate & $\begin{array}{l}\mathbf{N}_{R} \mathbf{N}_{C}^{-1} \mathbf{T}^{-1}, \text { or } \\
\mathbf{M}_{\mathrm{R}} \mathbf{M}_{\mathrm{C}^{-1}} \mathbf{T}^{-1}\end{array}$ \\
\hline \multirow{4}{*}{$\begin{array}{l}\text { Resource } \\
\text { patch level }\end{array}$} & $T$ & resource timescale (patch duration) & $\mathrm{T}$ \\
\hline & $L$ & resource length scale (distance between patches) & $\mathbf{L}$ \\
\hline & $R$ & characteristic resource density within patches & $\mathbf{N}_{R} \mathbf{L}^{-2}$ or $\mathbf{M}_{R} \mathbf{L}^{-2}$ \\
\hline & $C$ & characteristic consumer density within patches & $\mathbf{N}_{C} \mathbf{L}^{-2}$ or $\mathbf{M}_{\mathrm{C}} \mathbf{L}^{-2}$ \\
\hline \multirow[t]{5}{*}{ Timescales } & $T$ & resource patch duration timescale & $\mathrm{T}$ \\
\hline & $T_{\text {search }}=\frac{L^{2}}{s^{2} \tau}$ & consumer search timescale (diffusive movement) & $\mathrm{T}$ \\
\hline & $T_{\text {search }}=\frac{L}{S}$ & consumer search timescale (directed movement) & $\mathrm{T}$ \\
\hline & $T_{\text {repro. }}=\sigma$ & consumer reproduction timescale & $\mathrm{T}$ \\
\hline & $T_{\text {cons. }}=\frac{R}{\eta C}$ & timescale of resource consumption & $\mathrm{T}$ \\
\hline
\end{tabular}


To determine which consumer-resource pairs were dynamically similar, we performed a cluster analysis based on the Euclidean distance between the $\log _{10} \mathrm{Fr}$, Str, and Le ratios for each consumer-resource pair with complete agglomeration. The dendrogram was partitioned into five discrete clusters using a k-medioids algorithm (Reynolds et al. 2006; Schubert \& Rousseeuw 2019) within the ComplexHeatmap R package (Gu et al. 2016). This number of clusters was chosen based on the "elbow method" (Thorndike 1953, Figure S2) and our subjective assessment of the level of clustering that generated biologically meaningful groups. Within each cluster, we examined each interaction's ecosystem, consumer type, patch mobility, and consumer/resource size ratio, to explore whether these characteristics were associated with particular types of consumer-resource dynamics. All data and associated code for this project are available at https://github.com/allicramer/EcologicalPatchiness.

\section{Results and Discussion}

We analyzed a total of 33 interactions between consumers and their resource patches. Of these, 12 took place in terrestrial environments, 18 in marine environments, and 3 in freshwater (Table S1). The consumers ranged in average body size from $0.5 \mu \mathrm{m}$ to $22 \mathrm{~m}$, and in mass from 10 picograms to 5 tons. They included representatives of multiple trophic strategies (11 herbivores, 17 carnivores, and 5 detritivores) and body plans ( 2 microbes, 13 invertebrates, and 18 vertebrates). In most cases resource patches were composed of more than one organism; these covered a similarly large range of body sizes (Figure 3a). The resource patches lasted anywhere from 5 minutes (groups of seals swimming from their haul-out to deep water) to 1500 years (whale falls being buried by sediment), and were separated by distances ranging from 4 $\mathrm{cm}$ (nutrient patches from lysing phytoplankton) to $55 \mathrm{~km}$ (schools of zooplankton and fish in the open ocean) (Figure 3b). When the consumers' search, reproduction, and patch-depletion times were normalized by the durations of their resource patches, the resulting Frost, Strathmann, and Lessard numbers ranged across more than 10 orders of magnitude (Table 3, Figure 4). Despite the huge diversity of biology and range of scales considered, the dimensional analysis framework allowed us to identify a number of generalities, trends, and similarities across all the interactions. 
Table 3. Rates and dimensionless ratios for all consumer-resource pairs. Parameters are defined in Table 2; see Table S1 for a more detailed version of this table with references. Consumer and resource quantities are presented in their original units from the primary literature, which vary from interaction to interaction. For instance, the "currency" of an interaction could be expressed in individuals or biomass, densities could be areal or volumetric, etc. However, when plugged into the equations in Table 2, the quantities within each row are dimensionally consistent.

Abbreviations for units: $m=$ meters, $g=$ grams, $s=$ seconds, $\min =$ minutes, $h=$ hours,$d=$ days, $m o=$ months, $y=$ years, ha $=$ hectares, $c=$ consumer individuals, $r=$ resource individuals, $\mathrm{mol}=$ molecules, $\mathrm{pch}=$ patch, diff $=$ diffusive, dir $=$ directed .

\begin{tabular}{|c|c|c|c|c|c|c|c|c|c|c|c|c|c|c|c|c|c|}
\hline \multirow[b]{2}{*}{ ID } & \multirow[b]{2}{*}{ Consumer-resource pair } & \multicolumn{4}{|c|}{ Consumer level } & \multicolumn{4}{|c|}{ Resource patch level } & \multicolumn{4}{|c|}{ Timescales } & \multicolumn{4}{|c|}{ Dimensionless ratios } \\
\hline & & $s$ & $\tau$ & $\sigma$ & $\eta$ & c & $T(\mathrm{~s})$ & $L(\mathrm{~m})$ & $R$ & \begin{tabular}{|l|}
$\begin{array}{l}T_{\text {search }} \\
\text { (diff, s) }\end{array}$ \\
\end{tabular} & $\begin{array}{l}T_{\text {search }} \\
(\text { dir, s) }\end{array}$ & $\begin{array}{l}T_{\text {Tepro. }} \\
\text { (s) }\end{array}$ & $\begin{array}{l}T_{\text {cons. }} \\
\text { (s) }\end{array}$ & $\operatorname{Fr}$ (diff) & $\mathrm{Fr}$ (dir) & Str & Le \\
\hline 1 & Vibrio-Nutrients & $0.01 \mathrm{~mm} \mathrm{~s}^{-1}$ & $4 \mathrm{~s}$ & $5 \mathrm{~h}$ & $2.0 \times 10^{4} \mathrm{~mol} \mathrm{c}^{-1} \mathrm{~s}^{-1}$ & $5290 \mathrm{c} \mathrm{mm}^{-3}$ & 6000 & 0.001 & $3.0 \times 10^{20} \mathrm{~mol} \mathrm{~mL}^{-1}$ & $2.5 \times 10^{3}$ & 100 & $1.8 \times 10^{4}$ & $2.8 \times 10^{9}$ & 2.4 & 60.0 & $3.3 \times 10^{-1}$ & $2.1 \times 10^{-6}$ \\
\hline 2 & Pseudoalteromonas-Nutrients & $8.7 \times 10^{-5} \mathrm{~m} \mathrm{~s}^{-1}$ & $0.25 \mathrm{~s}$ & $3 \mathrm{~h}$ & & $1.0 \times 10^{4} \mathrm{c} \mathrm{mL}^{-1}$ & 900 & 0.04 & $5.7 \times 10^{-4} \mathrm{~mol} \mathrm{~L}^{-1}$ & $8.5 \times 10^{5}$ & 460 & $1.1 \times 10^{4}$ & 900 & $1.1 \times 10^{-3}$ & 2.0 & $8.3 \times 10^{-2}$ & 1.0 \\
\hline 3 & Bosmina-Diatoms & $0.8 \mathrm{~mm} \mathrm{~s}^{-1}$ & $6.7 \mathrm{~s}$ & $12.5 \mathrm{~h}$ & $168 \mathrm{r} \mathrm{c}^{-1} \mathrm{~d}^{-1}$ & $252 \mathrm{c} \mathrm{m}^{-3}$ & $8.6 \times 10^{4}$ & 1000 & $21 \mathrm{r} \mathrm{mL}^{-1}$ & $2.3 \times 10^{11}$ & $1.3 \times 10^{6}$ & $4.5 \times 10^{4}$ & $4.3 \times 10^{7}$ & $3.7 \times 10^{-7}$ & $6.9 \times 10^{-2}$ & 1.9 & $2.0 \times 10^{-3}$ \\
\hline 4 & Copepods-Phytoplankton layers & $1.42 \mathrm{~mm} \mathrm{~s}^{-1}$ & $1.25 \mathrm{~s}$ & $20 \mathrm{~d}$ & $4.0 \times 10^{4} \mathrm{rc}^{-1} \mathrm{~d}^{-1}$ & $2.0 \times 10^{4} \mathrm{c} \mathrm{m}^{-3}$ & $6.1 \times 10^{5}$ & 4000 & $1000 \mathrm{r} \mathrm{mL}^{-1}$ & $6.4 \times 10^{12}$ & $2.8 \times 10^{6}$ & $1.7 \times 10^{6}$ & $1.1 \times 10^{5}$ & $9.5 \times 10^{-8}$ & $2.2 \times 10^{-1}$ & $3.5 \times 10^{-1}$ & 5.6 \\
\hline 5 & Fruit flies-Marula fruit & $30 \mathrm{~cm} \mathrm{~s}^{-1}$ & $1 \mathrm{~s}$ & $15 \mathrm{~d}$ & $2 \times 10^{-6} \mathrm{~g} \mathrm{c}^{-1} \mathrm{~d}^{-1}$ & $1.0 \times 10^{4} \mathrm{cpch}^{-1}$ & $17.8 \times 10^{6}$ & 146 & $14 \mathrm{~kg} \mathrm{pch}^{-1}$ & $2.4 \times 10^{5}$ & 490 & $1.3 \times 10^{6}$ & $6.1 \times 10^{10}$ & 33 & $1.6 \times 10^{4}$ & 6.0 & $1.3 \times 10^{-4}$ \\
\hline 6 & Caterpillar-Kale plants & $0.2 \mathrm{~cm} \mathrm{~s}^{-1}$ & $6 \mathrm{~min}$ & $32 \mathrm{~d}$ & $0.41 \mathrm{~g} \mathrm{c}^{-1} \mathrm{~min}^{-1}$ & $100 \mathrm{c} \mathrm{pch}^{-1}$ & $1.3 \times 10^{7}$ & 1 & $100 \mathrm{~g} \mathrm{pch}^{-1}$ & 690 & 500 & $2.8 \times 10^{6}$ & 150 & $1.9 \times 10^{4}$ & $2.6 \times 10^{4}$ & 4.7 & $8.8 \times 10^{4}$ \\
\hline 7 & Caddisfly-Periphyton & $50 \mathrm{~cm} \mathrm{~min}^{-1}$ & $15 \mathrm{~s}$ & $1 \mathrm{y}$ & $3.3 \times 10^{-7} \mathrm{~g} \mathrm{c}^{-1} \mathrm{~h}^{-1}$ & $70 \mathrm{c} \mathrm{m}^{-2}$ & $1.1 \times 10^{7}$ & 0.19 & $1.2 \times 10^{-5} \mathrm{~g} \mathrm{~cm}^{-2}$ & 35 & 23 & $3.2 \times 10^{7}$ & $1.9 \times 10^{7}$ & $3.0 \times 10^{5}$ & $4.6 \times 10^{5}$ & $3.3 \times 10^{-1}$ & $5.5 \times 10^{-1}$ \\
\hline 8 & Bone worms-Whale falls & $10 \mathrm{~cm} \mathrm{~s}^{-1}$ & $22.2 \mathrm{~h}$ & $2 \mathrm{mo}$ & & $10 \mathrm{c} \mathrm{cm}^{-2}$ & $4.7 \times 10^{10}$ & $1.0 \times 10^{4}$ & & $1.3 \times 10^{5}$ & $1.0 \times 10^{5}$ & $5.2 \times 10^{6}$ & $3.2 \times 10^{8}$ & $3.8 \times 10^{5}$ & $4.7 \times 10^{5}$ & $9.1 \times 10^{3}$ & 150 \\
\hline 9 & Green crabs-Cockles & $0.54 \mathrm{~cm} \mathrm{~s}^{-1}$ & $3.2 \mathrm{~s}$ & $3 y$ & $6.5 \mathrm{r} \mathrm{c}^{-1} \mathrm{~d}^{-1}$ & $25 \mathrm{c} \mathrm{m}^{-2}$ & $6.5 \times 10^{6}$ & 200 & $1 \times 10^{4} \mathrm{r} \mathrm{m}^{-2}$ & $4.3 \times 10^{8}$ & $3.7 \times 10^{4}$ & $9.5 \times 10^{7}$ & $5.3 \times 10^{6}$ & $1.5 \times 10^{-2}$ & 170 & $6.9 \times 10^{-2}$ & 1.2 \\
\hline 10 & Desert locust-Vegetati & $10 \mathrm{~km} \mathrm{~h}^{-1}$ & $2 \mathrm{~s}$ & $60 \mathrm{~d}$ & $2 \mathrm{~g} \mathrm{c}^{-1} \mathrm{~d}^{-1}$ & $6.0 \times 10^{7} \mathrm{ckm}^{-2}$ & $1.6 \times 10^{7}$ & 10 & $1000 \mathrm{~kg} \mathrm{ha}^{-1}$ & 6.5 & 3.6 & $5.2 \times 10^{6}$ & $7.2 \times 10^{4}$ & $2.4 \times 10^{6}$ & $4.3 \times 10^{6}$ & 3.0 & 220 \\
\hline 11 & Dung beetle-Dung & $0.05 \mathrm{~m} \mathrm{~s}^{-1}$ & $8 \mathrm{~s}$ & $6 \mathrm{mo}$ & $0.91 \mathrm{~g} \mathrm{c}^{-1} \mathrm{~min}^{-1}$ & $1 \mathrm{cpch}^{-1}$ & $8.7 \times 10^{6}$ & 32 & $5620 \mathrm{~g} \mathrm{pch}^{-1}$ & $5.1 \times 10^{4}$ & 640 & $1.6 \times 10^{7}$ & $3.7 \times 10^{5}$ & 170 & $1.4 \times 10^{4}$ & $5.6 \times 10^{-1}$ & 24.0 \\
\hline 12 & Ctenophores-Copepods & $0.7 \mathrm{~cm} \mathrm{~s}^{-1}$ & $10 s$ & $7.75 d$ & $10 \mathrm{r} \mathrm{c}^{-1} \mathrm{~h}^{-1}$ & $50 \mathrm{c} \mathrm{m}^{-3}$ & $4.3 \times 10^{5}$ & 4000 & $2 \times 10^{4} \mathrm{r} \mathrm{m}^{-3}$ & $3.3 \times 10^{10}$ & $5.7 \times 10^{5}$ & $6.7 \times 10^{5}$ & $1.4 \times 10^{5}$ & $1.3 \times 10^{-5}$ & $7.6 \times 10^{-1}$ & $6.5 \times 10^{-1}$ & 3.0 \\
\hline 13 & Beach mouse-Dune seeds & $11.4 \mathrm{~km} \mathrm{~h}^{-1}$ & $1 \mathrm{~s}$ & $26 \mathrm{~d}$ & $14 \mathrm{r} \mathrm{c}^{-1} \mathrm{~d}^{-1}$ & $8 \mathrm{cha}^{-1}$ & $3.2 \times 10^{9}$ & 15 & $270 \mathrm{r} \mathrm{m}^{-2}$ & 22 & 4.7 & $2.3 \times 10^{6}$ & $2.1 \times 10^{9}$ & $1.4 \times 10^{8}$ & $6.7 \times 10^{8}$ & $1.4 \times 10^{3}$ & 1.5 \\
\hline 14 & Chimney swifts-Aerial plankton & $11.3 \mathrm{~m} \mathrm{~s}^{-1}$ & $2 \mathrm{~s}$ & $2 y$ & $415 \mathrm{r} \mathrm{c}^{-1} \mathrm{~h}$ & $3.8 \times 10^{-6} \mathrm{c} \mathrm{m}^{-3}$ & 3600 & $2.5 \times 10^{4}$ & $0.001 \mathrm{r} \mathrm{m}^{-3}$ & $2.5 \times 10^{6}$ & $2.2 \times 10^{3}$ & $6.3 \times 10^{7}$ & $2.3 \times 10^{3}$ & $1.5 \times 10^{-3}$ & 1.6 & $5.7 \times 10^{-5}$ & 1.6 \\
\hline 15 & Northern sea stars-Mussels & $5 \mathrm{~cm} \mathrm{~min}^{-1}$ & $10 \mathrm{~min}$ & $2 y$ & $2 \mathrm{rc}^{-1} \mathrm{~d}^{-1}$ & $5.5 \mathrm{c} \mathrm{m}^{-2}$ & $2.2 \times 10^{8}$ & 40 & $3000 \mathrm{r} \mathrm{m}^{-2}$ & $3.8 \times 10^{6}$ & $4.8 \times 10^{4}$ & $6.3 \times 10^{7}$ & $3.2 \times 10^{7}$ & 57.5 & $4.6 \times 10^{3}$ & 3.5 & 7.0 \\
\hline 16 & Trout-Zooplankton & $30 \mathrm{~cm} \mathrm{~s}^{-1}$ & $7 \mathrm{~s}$ & $4 y$ & $0.02 \mathrm{~g} \mathrm{c}^{-1} \mathrm{~s}^{-1}$ & $3.5 \times 10^{-3} \mathrm{c} \mathrm{m}^{-3}$ & $1.1 \times 10^{4}$ & 1000 & $50 \mathrm{~g} \mathrm{~m}^{-3}$ & $1.6 \times 10^{6}$ & $3.3 \times 10^{3}$ & $1.3 \times 10^{8}$ & $7.1 \times 10^{5}$ & $6.8 \times 10^{-3}$ & 3.2 & $8.6 \times 10^{-5}$ & $1.5 \times 10^{-2}$ \\
\hline 17 & Red knots-Cockles & $20 \mathrm{~m} \mathrm{~s}^{-1}$ & $40 \mathrm{~s}$ & $2 y$ & $0.4 \mathrm{mg} \mathrm{c}^{-1} \mathrm{~s}^{-1}$ & $1 \times 10^{-4} \mathrm{c} \mathrm{m}^{-2}$ & $2.2 \times 10^{4}$ & $1.0 \times 10^{4}$ & $2 \mathrm{~g} \mathrm{~m}^{-2}$ & $6.3 \times 10^{3}$ & 500 & $6.3 \times 10^{7}$ & $5.0 \times 10^{7}$ & 3.5 & 43 & $3.4 \times 10^{-4}$ & $4.3 \times 10^{-4}$ \\
\hline 18 & Red knots-Clams & $20 \mathrm{~m} \mathrm{~s}^{-1}$ & $40 \mathrm{~s}$ & $2 y$ & $0.4 \mathrm{mg} \mathrm{c}^{-1} \mathrm{~s}^{-1}$ & $1 \times 10^{-4} \mathrm{c} \mathrm{m}^{-2}$ & $2.2 \times 10^{4}$ & 1000 & $1 \mathrm{~g} \mathrm{~m}^{-2}$ & 63 & 50 & $6.3 \times 10^{7}$ & $2.5 \times 10^{7}$ & 35 & 430 & $3.4 \times 10^{-4}$ & $8.6 \times 10^{-4}$ \\
\hline 19 & Terns-Forage fishes & $11 \mathrm{~m} \mathrm{~s}^{-1}$ & $10 \mathrm{~s}$ & $4 y$ & $40 \mathrm{r} \mathrm{c}^{-1} \mathrm{~d}^{-1}$ & $0.05 \mathrm{c} \mathrm{m}^{-2}$ & $2.2 \times 10^{4}$ & 5000 & $100 \mathrm{rm}^{-2}$ & $2.1 \times 10^{4}$ & 46 & $1.3 \times 10^{8}$ & $4.3 \times 10^{6}$ & 1.1 & 48 & $1.7 \times 10^{-4}$ & $5.0 \times 10^{-3}$ \\
\hline 20 & Pisaster sea star-Mussels & $1.67 \mathrm{~m} \mathrm{~h}^{-1}$ & $10 \mathrm{~min}$ & $6 y$ & $80 \mathrm{r} \mathrm{c}^{-1} \mathrm{y}^{-1}$ & $16.5 \mathrm{c} \mathrm{m}^{-2}$ & $2.9 \times 10^{8}$ & 250 & $250 \mathrm{rm}^{-2}$ & $4.8 \times 10^{8}$ & $5.4 \times 10^{5}$ & $1.9 \times 10^{8}$ & $6.0 \times 10^{6}$ & 0.59 & 530 & 1.5 & 48 \\
\hline 21 & Eastern gray squirrel-Acorn & $50 \mathrm{~cm} \mathrm{~s}^{-1}$ & $10 \mathrm{~s}$ & $1.25 y$ & $6.1 \times 10^{-3} \mathrm{r} \mathrm{c}^{-1} \mathrm{~s}^{-1}$ & $0.03 \mathrm{c} \mathrm{pch}^{-1}$ & $7.8 \times 10^{6}$ & 8.3 & $1000 \mathrm{rpch}^{-1}$ & 28 & 17 & $3.9 \times 10^{7}$ & $2.5 \times 10^{4}$ & $2.8 \times 10^{5}$ & $4.7 \times 10^{5}$ & 0.2 & 310 \\
\hline 22 & Murres-Zooplankton/fish & $20.9 \mathrm{~m} \mathrm{~s}^{-1}$ & $1 \mathrm{~min}$ & $4 y$ & $700 \mathrm{r} \mathrm{c}^{-1} \mathrm{~d}^{-1}$ & $26 \mathrm{ckm}^{-2}$ & $2.9 \times 10^{4}$ & $5.5 \times 10^{4}$ & $75 \mathrm{r} \mathrm{m}^{-2}$ & $1.2 \times 10^{5}$ & $2.6 \times 10^{3}$ & $1.3 \times 10^{8}$ & $3.6 \times 10^{8}$ & $2.5 \times 10^{-1}$ & 11 & $2.3 \times 10^{-4}$ & $8.1 \times 10^{-5}$ \\
\hline 23 & Penguin-Fish school & $1.1 \mathrm{~m} \mathrm{~s}^{-1}$ & $1 \mathrm{~min}$ & $2.5 \mathrm{y}$ & $192 \mathrm{~g} \mathrm{c}^{-1} \mathrm{~d}^{-1}$ & $4 \mathrm{c} \mathrm{pch}^{-1}$ & $5.0 \times 10^{4}$ & 250 & $243 \mathrm{~kg} \mathrm{pch}^{-1}$ & 860 & 230 & $7.9 \times 10^{7}$ & $2.7 \times 10^{7}$ & 59 & 220 & $6.4 \times 10^{-4}$ & $1.9 \times 10^{-3}$ \\
\hline 24 & Spider monkeys-Fruit trees & $4.8 \mathrm{~m} \mathrm{~min}^{-1}$ & $5 \mathrm{~min}$ & $6.5 \mathrm{y}$ & & & $6.0 \times 10^{5}$ & 45.8 & & $1.1 \times 10^{3}$ & 700 & $2.0 \times 10^{8}$ & 810 & 550 & 860 & $3.0 \times 10^{-3}$ & 750 \\
\hline 25 & Beaver-Maple tree & $28 \mathrm{~m} \mathrm{~min}^{-1}$ & $1 \mathrm{~min}$ & $5.4 \mathrm{y}$ & $6.7 \times 10^{-3} \mathrm{rc}^{-1} \mathrm{~s}$ & $2.6 \mathrm{c}$ & $6.3 \times 10^{9}$ & 50 & $0.18 \mathrm{r} \mathrm{m}^{-2}$ & 19 & 70 & $1.7 \times 10^{8}$ & $3.8 \times 10^{7}$ & $3.3 \times 10^{7}$ & $9.0 \times 10^{7}$ & 37.0 & 170 \\
\hline 26 & Mongolian gazelle-Vegetation & $8.2 \mathrm{~km} \mathrm{~d}^{-1}$ & $1 \mathrm{~d}$ & $2 y$ & $5 \mathrm{~g} \mathrm{c}^{-1} \mathrm{~min}^{-1}$ & $281 \mathrm{c} \mathrm{km}^{-2}$ & 2. & $2.0 \times 10^{4}$ & $20 \mathrm{~g} \mathrm{~m}^{-2}$ & $5.1 \times 10^{5}$ & $2.1 \times 10^{5}$ & $6.3 \times 10^{7}$ & $8.5 \times 10^{5}$ & 5.0 & 12 & $10^{-2}$ & 3.0 \\
\hline 27 & Wolf-Caribou & $8.7 \mathrm{~km} \mathrm{~h}^{-1}$ & $1 \mathrm{~h}$ & $22 \mathrm{mo}$ & $0.045 \mathrm{r} \mathrm{c}^{-1} \mathrm{~d}^{-1}$ & $9 \mathrm{cpch}^{-1}$ & $2.3 \times 10^{7}$ & $2.5 \times 10^{5}$ & $1000 \mathrm{r} \mathrm{pch}^{-1}$ & $3.0 \times 10^{6}$ & $6.1 \times 10^{5}$ & $5.7 \times 10^{7}$ & $2.1 \times 10^{8}$ & 7.9 & 39 & $4.1 \times 10^{-1}$ & $1.1 \times 10^{-1}$ \\
\hline 28 & Fur seals-Pollock & $3.5 \mathrm{~m} \mathrm{~s}^{-1}$ & $1 \mathrm{~min}$ & $4 y$ & $3 \mathrm{~kg} \mathrm{c}^{-1} \mathrm{~d}^{-1}$ & $2 \mathrm{cpch}^{-1}$ & $4.3 \times 10^{4}$ & 300 & $3 \mathrm{~kg} \mathrm{pch}^{-1}$ & 120 & 86 & $1.3 \times 10^{8}$ & $4.3 \times 10^{4}$ & 350 & 500 & $3.4 \times 10^{-4}$ & 1.0 \\
\hline 29 & Elk-Forest patch & $759 \mathrm{~m} \mathrm{~h}^{-1}$ & $3 \mathrm{~min}$ & $5.7 \mathrm{y}$ & $989 \mathrm{~g} \mathrm{c}^{-1} \mathrm{~d}^{-1}$ & $0.625 \mathrm{c} \mathrm{km}^{-2}$ & $1.1 \times 10^{10}$ & 427 & $245 \mathrm{~g} \mathrm{~m}^{-2}$ & $2.3 \times 10^{4}$ & $2.0 \times 10^{3}$ & $1.8 \times 10^{8}$ & $3.4 \times 10^{10}$ & $4.9 \times 10^{5}$ & $5.5 \times 10^{6}$ & 62.0 & $3.3 \times 10^{-1}$ \\
\hline 30 & Tuna-Herring & $1.63 \mathrm{~m} \mathrm{~s}^{-1}$ & $1.8 \mathrm{~min}$ & $14 \mathrm{y}$ & $24.6 \mathrm{r} \mathrm{c}^{-1} \mathrm{~d}^{-1}$ & $0.54 \mathrm{c} \mathrm{m}^{-2}$ & $4.3 \times 10^{4}$ & $3.3 \times 10^{4}$ & $10 \mathrm{r} \mathrm{m}^{-2}$ & $3.8 \times 10^{6}$ & $2.0 \times 10^{4}$ & $4.4 \times 10^{8}$ & $6.5 \times 10^{4}$ & $1.1 \times 10^{-2}$ & 2.1 & $9.8 \times 10^{-5}$ & $6.6 \times 10^{-1}$ \\
\hline 31 & White sharks-Seals & $1.34 \mathrm{~m} \mathrm{~s}^{-1}$ & $30 \mathrm{~s}$ & $12.3 y$ & $0.25 \mathrm{r} \mathrm{c}^{-1} \mathrm{~d}^{-1}$ & $1 \mathrm{cpch}^{-1}$ & 300 & 70 & $8 \mathrm{rpch}^{-1}$ & 91 & 52 & $3.9 \times 10^{8}$ & $2.8 \times 10^{6}$ & 3.3 & 5.7 & $7.7 \times 10-07$ & $1.1 \times 10^{-4}$ \\
\hline 32 & Killer whale-Chinook salmon & $1.6 \mathrm{~m} \mathrm{~s}^{-1}$ & $1 \mathrm{~min}$ & $24 \mathrm{y}$ & $7 \mathrm{r} \mathrm{c}^{-1} \mathrm{~min}^{-1}$ & $1.2 \times 10^{-6} \mathrm{c} \mathrm{m}^{-3}$ & 1200 & 500 & $0.05 \mathrm{n} \mathrm{m}^{-3}$ & $1.6 \times 10^{3}$ & 310 & $7.6 \times 10^{8}$ & $3.6 \times 10^{5}$ & $7.4 \times 10^{-1}$ & 3.8 & $1.6 \times 10^{-6}$ & $3.4 \times 10^{-3}$ \\
\hline 33 & Blue whale-Krill & $3.7 \mathrm{~km} \mathrm{~h}^{-1}$ & $400 \mathrm{~s}$ & $10 y$ & $105 \mathrm{~kg} \mathrm{c}^{-1} \mathrm{~min}^{-1}$ & $0.02 \mathrm{c} \mathrm{km}^{-2}$ & $2.2 \times 10^{4}$ & 7000 & $1 \mathrm{~kg} \mathrm{~m}^{-2}$ & $1.2 \times 10^{5}$ & $6.8 \times 10^{3}$ & $3.2 \times 10^{8}$ & $2.7 \times 10^{7}$ & $1.9 \times 10^{-1}$ & 3.2 & $6.9 \times 10^{-5}$ & $7.9 \times 10^{-4}$ \\
\hline
\end{tabular}



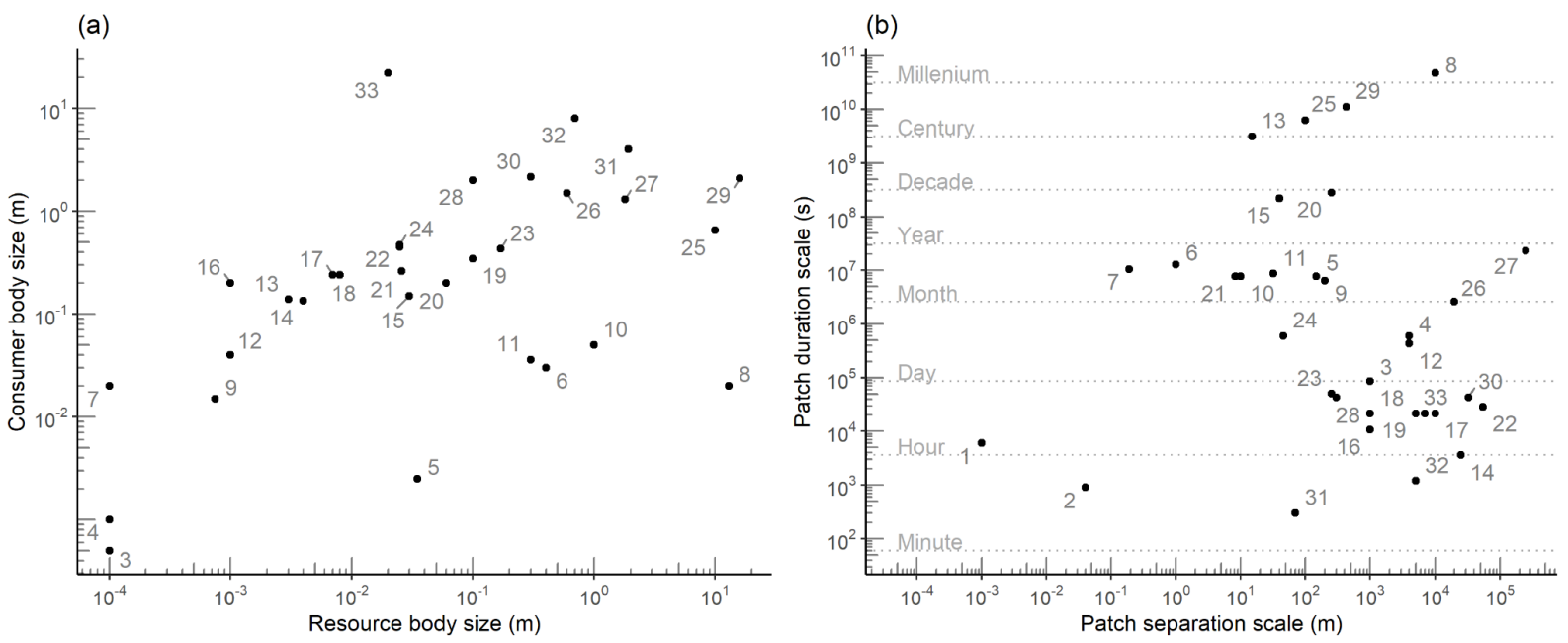

Figure 3. The sizes of consumer and resource organisms, and the spatio-temporal scales of resource patches, spanned many orders of magnitude. (a) Body size of consumer organisms (y-axis) plotted against the size of an individual resource item. Numbered labels refer to the interaction IDs in Table 3 , each containing a consumer organism and a corresponding resource (Note that the two bacterial interactions are excluded, since "organism size" is poorly defined for a dissolved nutrient resource). (b) Resource patches spanned approximately eight orders of magnitude in their spatial and temporal scales. Each point represents the resource patch within an interaction, labeled as in (a). The horizontal axis represents the typical distance scale separating patches in space, ranging from $\sim 1 \mathrm{~mm}$ (microscale nutrient plumes, interaction 1) to $\sim 250 \mathrm{~km}$ (caribou herds, interaction 27 ). The vertical axis represents the patches' typical duration, ranging from $\sim 5 \mathrm{~min}$ (seal groups, interaction 31 ) to $\sim 1,500$ years (whale falls, 8).

\section{All interactions were functionally patchy}

Every consumer-resource interaction considered here was functionally patchy with respect to movement, population growth, or consumption. All interactions had at least one of the three dimensionless ratios greater than 1, and in 25 out of the 33 pairs, at least one of the ratios was $>10$ (Table 3 ). Seven of the 33 consumer-resource interactions $(21 \%)$ had all three numbers > 1 , indicating that patch dynamics had a significant influence on all of these aspects of consumer-resource ecology. Given this, a model using a strict mean-field assumption would not be justifiable for any of these interactions. While our deliberate selection of systems with patchy resources precludes generalizing this result to all consumer-resource interactions, it still stands as a warning to ecologists dealing with foraging movements, consumption/predation, or trophic transfer rates: if a system looks like it might have patchy dynamics, then they probably shouldn't be ignored. 
Even though patch dynamics could not be ignored entirely in any of the consumer-resource pairs, the majority (26 out of 33 interactions, or $79 \%$ ) had at least one dimensionless ratio $<1$, indicating that at least one of the processes (movement, reproduction, or consumption) could be simplified using a mean-field approximation. As a concrete example, consider interaction 19, between common terns Sterna hirundo and forage fishes. Terns prey on schools of small fishes forced to the surface by underwater predators (Safina \& Burger 1985) or physical flows (Urmy \& Warren 2018). Surface schools are typically separated by several $\mathrm{km}$ and remain available for up to a few hours. This interaction is characterized by a $\mathrm{Fr}_{\text {dir }}$ of 48 , indicating that the terns' rapid flight allows them to spend more time over fish patches than over empty water. When modeling trophic transfer between fishes and terns, estimates of energy flow based on the mean prey density (e.g., measured by a fishery survey) would be biased low. A more reasonable estimate would be parameterized based on the mean prey density within schools, perhaps with second-order effects to account for search times and other foraging dynamics. At the same time, terns visit many patches per generation ( $\operatorname{Str}=1.7 \times 10^{-4}$ ), so if a parameterized energy transfer function were developed, averaging it over days, weeks, or even an entire breeding season would be justifiable. Finally, the low Lessard number ( $\mathrm{Le}=0.005$ ) indicates that terns rarely deplete patches, so models of this interaction can treat the density and distribution of their prey as endogenous variables (cf. Urmy 2021).

Analogous simplifications can be identified for most of the consumer-resource interactions (Table 4). In general, low values of the Frost number indicate that spatial mean-field models may be appropriate, while high values mean they are not. As always, spatial mean-field models are highly dependent on scale; interactions with a high Frost number may be modeled based on mean densities inside patches. Similarly, low values of the Strathmann number suggest temporal averaging may be acceptable. With Str $>>1$, many generations of consumers can reproduce in each patch and the appropriateness of mean-field models depends on the impacts of those generations (i.e., Le). If the consumers do not deplete the patches (i.e., Le $<<1$ ), the interaction is essentially a traditional metapopulation (Hanski 1998). On the other hand, if Le > 1 , there will be a more complex balance between patch appearance, colonization, and depletion, similar to the susceptible-infected-recovered dynamics familiar from epidemiological models (Kermack et al. 1927).

If any of the ratios are $\sim 1$, simple approximations overlook crucial aspects of the system. In these cases, the relevant dynamic process(es) must be modeled or parameterized explicitly. Dimensionless ratios such as Fr, Str, and Le provide a simple way to check whether complex models are required. Crucially, they also provide a means to develop those models in a more general way. Models expressed in terms of dimensionless ratios benefit from a reduced parameter space and often express a problem in its simplest possible form (Stephens and Dunbar 1993). Additionally, in such models the specific units attached to the variables are irrelevant and the essential dynamics of the system become clear. Connections between dynamically similar systems can be identified independent of their absolute size or scale. 
Table 4. Guidance for modelling approaches based on dimensionless ratios.

\begin{tabular}{|c|c|c|c|}
\hline & Ratio $<<1$ & Ratio 1 & Ratio $>1$ \\
\hline $\mathrm{Fr}$ & $\begin{array}{l}\text { Consumers spend most } \\
\text { time outside patches. } \\
\text { Encounter and trophic } \\
\text { transfer rates } \propto \text { average } \\
\text { densities of consumer and } \\
\text { resource over entire } \\
\text { region. Mean-field } \\
\text { assumption may be ok. }\end{array}$ & $\begin{array}{l}\text { Movement and foraging } \\
\text { behaviors must be modeled } \\
\text { or parameterized explicitly. }\end{array}$ & $\begin{array}{l}\text { Consumers spend most of } \\
\text { their time inside patches. } \\
\text { Encounter and trophic } \\
\text { transfer rates } \propto \text { average } \\
\text { densities of consumer and } \\
\text { resource within patches (by } \\
\text { definition, higher than } \\
\text { spatial average). }\end{array}$ \\
\hline Str & $\begin{array}{l}\text { Consumers visit many } \\
\text { patches per generation. } \\
\text { Temporal averaging ok. }\end{array}$ & $\begin{array}{l}\text { Multi-trophic population } \\
\text { dynamics à la 2-species } \\
\text { Lotka-Volterra, must be } \\
\text { modeled or parameterized } \\
\text { explicitly. }\end{array}$ & $\begin{array}{l}\text { Multiple generations } \\
\text { reproduce within each } \\
\text { patch. If Le }<<1 \text {, } \\
\text { approaches } \\
\text { metapopulation dynamics. }\end{array}$ \\
\hline Le & $\begin{array}{l}\text { Consumers have negligible } \\
\text { effect on duration or } \\
\text { density of resource } \\
\text { patches, which appear and } \\
\text { disappear independently of } \\
\text { consumers. Feedbacks } \\
\text { between consumer and } \\
\text { resource density can be } \\
\text { ignored. }\end{array}$ & \multicolumn{2}{|c|}{$\begin{array}{l}\text { Consumers may deplete resource patches. Effects of } \\
\text { movement, reproduction, dispersal, and/or death must be } \\
\text { modeled or parameterized to account for persistence of } \\
\text { populations. If Le }>>1 \text {, resource patches deplete quickly. }\end{array}$} \\
\hline
\end{tabular}

\section{Assessing Grünbaum's conjectures for organisms in patchy environments}

Across all interactions, the directed Frost and Strathmann numbers were positively correlated $(r$ $=0.68, p<0.001)$. Most interactions fell in the upper-right three quadrants in Fr-Str space (Figure 4a), indicating that consumers would be able to find and exploit patches through movement, reproduction, or both. For most consumers, the Frost number increased dramatically when directed rather than diffusive movement was assumed. Nine consumers would only be able to occupy patches if they used directed movement: if they relied on random search behavior, their theoretical search time would be longer than a patch's typical duration. Figure S1 gives an alternate visualization of critical Frost numbers following the scheme of Grünbaum's (2012) Figure 1a; this presentation suggests all consumers would be able to access their patches. Two consumer-resource pairs (ctenophores feeding on copepods, interaction 12, and copepods consuming phytoplankton thin layers, interaction 4) fell just inside the lower-left 
quadrant, suggesting they should be unable to locate and exploit their respective resources. Only one consumer (the water flea Bosmina feeding on phytoplankton) fell in the upper-left quadrant, indicating a primary reliance on explosive reproduction to maintain populations within patches. Finally, most of the large marine predators exploited patches that are too ephemeral to allow reproduction within them.

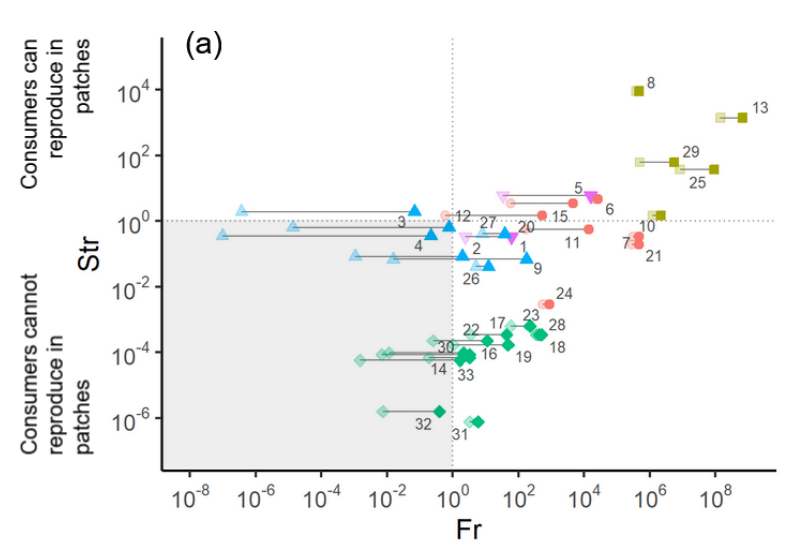

1. Vibrio - Nutrients

2. Pseudoalteromonas - Nutrients

3. Bosmina - Diatoms

4. Copepods - Phytoplankton

5. Fruit flies - Marula fruit

6. Caterpillar - Kale plants

7. Caddisfly - Periphyton

8. Bone worms - Whale falls

9. Green crabs - Cockles

10. Desert locust - Vegetation

11. Dung beetle - Dung

12. Ctenophores - Copepods

13. Beach mouse - Dune seeds

14. Chimney swifts - Aerial plankton

15. Northern sea stars - Mussels

16. Trout - Zooplankton

17. Red knots - Cockles

18. Red knots - Clams
19. Terns - Forage fishes

20. Pisaster sea star- Mussels

21. Eastern gray squirrel - Acorn

22. Murres - Zooplankton/fish

23. Penguin - Fish school

24. Spider monkeys - Fruit trees

25. Beaver - Maple tree

26. Mongolian gazelle - Vegetation

27. Wolf - Caribou

28. Fur seals - Pollock

29. Elk - Forest patch

30. Tuna - Herring

31. White shark - Seals

32. Killer whale - Chinook salmon

33. Blue whale - Krill
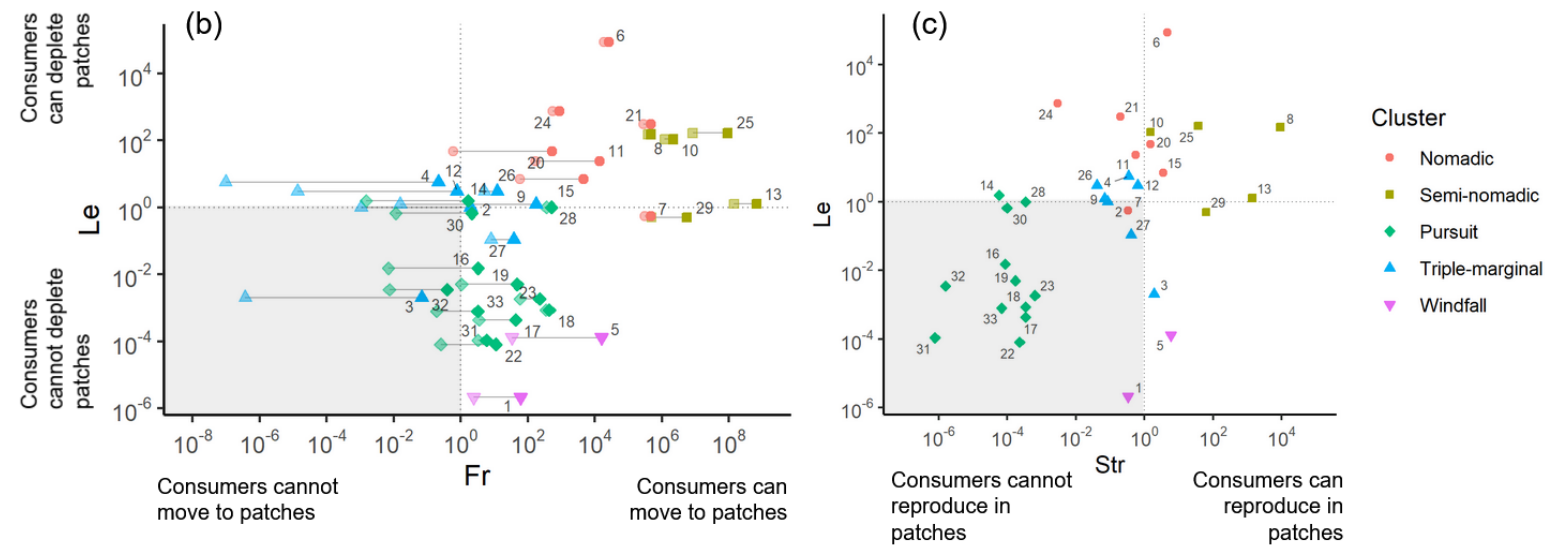

Figure 4 - All consumer-resource interactions plotted on logarithmic axes in dimensionless Frost-Strathmann (a), Frost-Lessard (b), or Strathmann-Lessard (c) space, showing the relative importance of movement ( $\mathrm{Fr}$ ), reproduction (Str), and depletion (Le) in driving patch dynamics.. In (a) and (b), two points, connected by a line, are plotted for each consumer-resource pair: the lighter point is the Frost number assuming diffusive (i.e. random walk) movement, while the filled point is the Frost number for directed movement. The horizontal and vertical grey lines show the critical values, where Fr, Str, and Le equal 1 . The shaded regions on each plot indicate interactions which are not functionally patchy with respect to each pair of dimensionless ratios. For interactions in these regions, the mean-field simplification may be (partially) justifiable. Point shapes and colors represent clusters of similar interactions identified via a k-medioids analysis; see Figure 5 and text for details.

As shown in Frost-Lessard space (Figure 4b), roughly half the consumers (18 out of 33, or 54\%) had Le $>1$ and were thus theoretically capable of depleting their resource patches. Of these patch-depleting consumers, all but two also had $\mathrm{Fr}>1$, suggesting they were able to effectively 
move between patches (Figure 4b). There was a positive correlation between Fr and Le in log-log space $(r=0.44, p=0.01)$, with relatively faster-moving consumers also tending to consume their resource patches faster. Only two consumer-resource pairs (copepodsphytoplankton thin layers and ctenophores-copepods) fell in the upper-left quadrant of Figure $4 \mathrm{~b}$, where depletion of patches is possible but movement between them is not.

Across all consumer-resource pairs, there was also a positive correlation between the Str and Le numbers $(r=0.48, p=0.004)$, indicating that the capacity for rapid population growth within a patch was associated with the ability to totally consume the resource (Figure 4c). However, consumer-resource pairs were present in all quadrants, so this tendency was far from a rule. Thirteen consumers fell in the upper-left quadrant (Str $<1$, Le $>1$ ), indicating they could deplete patches without being able to reproduce within them. Only three consumers--Bosmina, fruit flies, and elk--were capable of reproducing in a patch but incapable of depleting it, placing them in the lower-right quadrant with $\mathrm{Str}<1$ and Le $<1$. Consumer populations will only persist if they can locate patches easily, reproduce within patches, or both, leading Grünbaum to predict that consumers must have either Fr or Str $>1$. In our analysis, this prediction was borne out for 31 out of 33 interactions. The two exceptions were both planktonic consumers feeding on a planktonic resource: copepods - phytoplankton thin layers $\left(\mathrm{Fr}_{\mathrm{dir}}=0.2, \mathrm{Str}=0.35\right)$, and ctenophores - copepods $\left(\mathrm{Fr}_{\mathrm{dir}}=0.76, \mathrm{Str}=0.65\right)$. According to the logic of the dimensionless ratios, populations of these consumers should not be able to persist in nature--though this prediction is belied by the fact that ctenophores and copepods are two of the most widespread and abundant animal groups in the ocean. There are several possible explanations for this discrepancy. One is mismeasurement of the relevant patch scales or generation times: since both Frost and Strathmann numbers were within an order of magnitude of 1 , only relatively small errors would be required to move them from above to below the critical value. Another, perhaps more likely, explanation is our use of generation times, rather than intrinsic rates of population growth, to estimate the Strathmann number. Both copepods and ctenophores can produce more than one offspring per generation, so their potential for explosive growth in patches is higher than predicted by the generation time alone. The intrinsic rate of increase would be a more appropriate basis for calculating the Strathmann number and should be used in future studies. However, values for it were not available in the literature for many of the consumers considered here, so we used generation time instead. The only consumer-resource interaction that fell in the upper-left quadrant in Fr-Str space was Bosmina - diatoms, another planktonic pair. Whether or not a purely reproductive strategy for patch exploitation is viable in non-planktonic interactions would be an interesting topic for future research.

This dimensionless framework reveals the importance of understanding a consumer's movement ecology. Some consumers need directive search behavior to survive. Eight consumers would not be able to access their resource patches using diffusive search: white sharks, killer whales, chimney swifts, trout, common terns, common murres, green crabs, and the marine bacterium Pseudoalteromonas. It is striking that all but one of these (the green crab) feed on mobile prey in aquatic or aerial pelagic environments. This suggests that effective movement strategies are critical to the survival of these animals, and that this may be general to 
pelagic predators, whether they are pursuing prey through air or water. While we did not estimate costs of movement for consumers, the fact that none of these predators' Frost numbers were $>1$ is consistent with Grünbaum's conjecture that species will evolve movement abilities "good enough" to exploit their prey, but not greater.

\section{Dynamic similarities across scales and systems}

A number of dynamic similarities were revealed when interactions were clustered according to their dimensionless ratios. We identified five groups of dynamically similar consumer-resource interactions (Figure 5). These groups should not be over-interpreted, since the selection of consumer-resource pairs and the number of clusters were both chosen semi-subjectively. The clusters could thus potentially shift with the addition of new pairs. Still, these clusters provide a useful perspective on generalities in patch dynamics, and hint at some of the reasons for their similarities and differences.

The first cluster was defined by Fr $>1$, Str $\sim 1$, and Le $>1$, indicating consumers could move relatively easily between patches and sometimes reproduce within them, but usually depleted them first. We termed these interactions nomadic, since these dynamics require constant movement by the consumers. We termed the second cluster semi-nomadic, since it shared similar features with nomadic interactions, though with faster movement and reproduction relative to patch duration ( $\mathrm{Fr}>>1$, Str $>1$ ) and a slightly lower chance of depletion (Le $\sim 1)$.

Both of these clusters were grouped together in the same branch of the dendrogram, and their broadly similar patch dynamics can be seen as falling on a continuum, with stationary resources exploited by mostly-herbivorous consumers. Consumers within the semi-nomadic cluster had, on average, higher Frost and Strathmann numbers, indicating they could locate and reproduce within patches more easily than the nomads. The nomadic cluster included three benthic aquatic and four terrestrial interactions, while all interactions in the semi-nomadic cluster were terrestrial except for Osedax bone worms on whale falls. Taken together, these two clusters encompass interactions where consumers travel easily between patches, moving from one to the next as they deplete their stationary resources. This depletion could happen relatively quickly (e.g., a quarter of an hour for a troupe of spider monkeys to eat the fruit off a tree) or over multiple generations (e.g., the decade or so for a population of bone worms to decompose a whale skeleton). The main difference between nomadic and semi-nomadic clusters was thus the length of residency the consumers had within their stationary resource patches. 


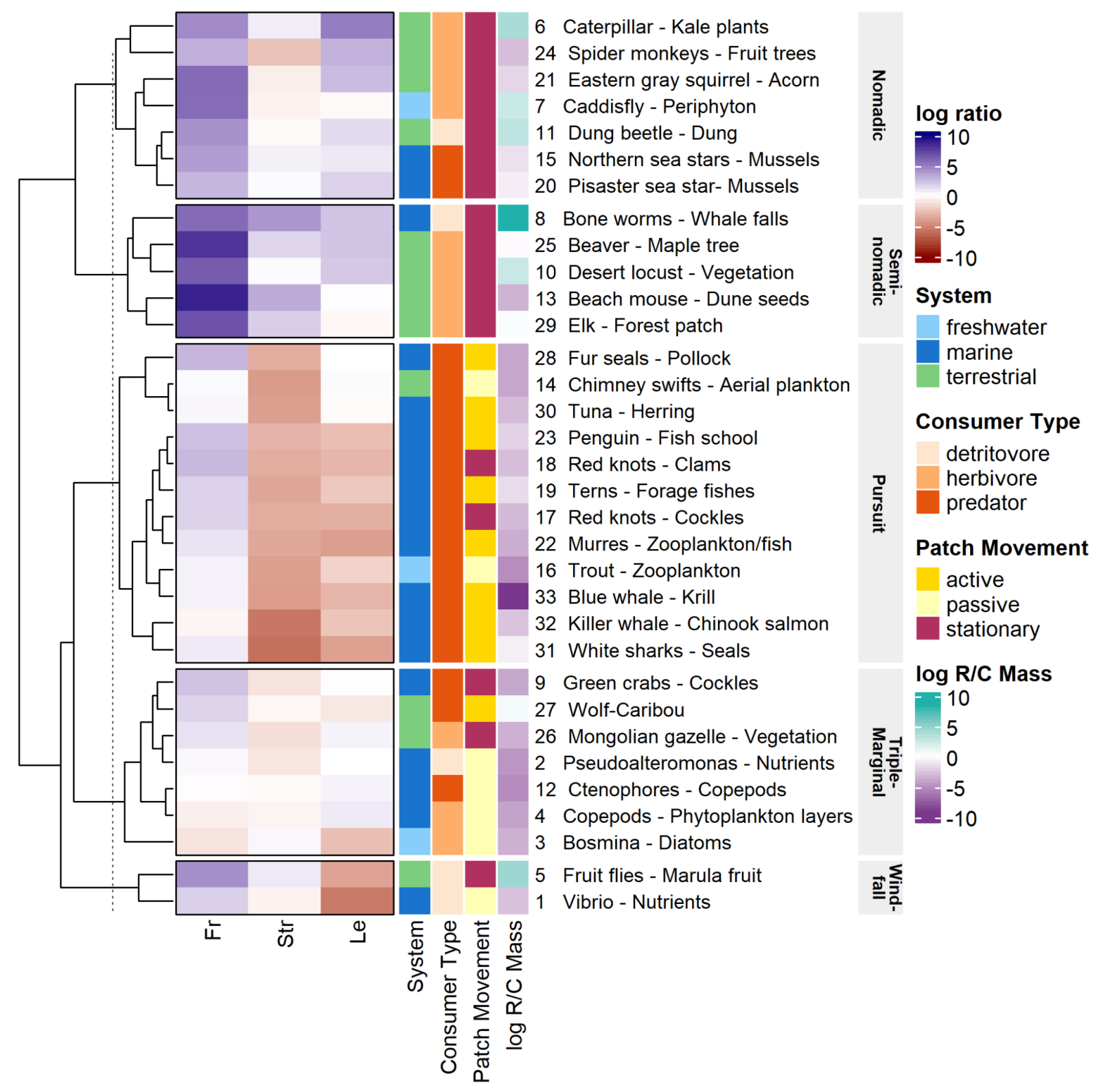

Figure 5. Clustering based on the log-transformed directed Frost, Strathmann, and Lessard numbers identified five groups of dynamically similar consumer-resource interactions. The three left columns in the heatmap (red-blue color scale) show the logarithmic values of $\mathrm{Fr}$, Str, and Le for each interaction. The four right columns display the ecosystem in which the interaction takes place, the consumer and patch types, and the log-ratio of body mass between resource $(R)$ and consumer $(C)$. These latter four variables are shown for additional context but were not included in the clustering, which was driven entirely by Fr, Str, and Le. Each cluster is labeled with a descriptive name; see text for details.

The third cluster included 12 interactions. All were driven by movement ( $\mathrm{Fr}>1)$, with very slow reproductive dynamics (Str $<<1$ ) and marginal to very weak depletion (Str $\sim 1$ to $\operatorname{Str}<<1$ ). 
Based on their movement-dominated dynamics, we termed these interactions pursuit-type. While these consumers still moved relatively rapidly between resource patches, their Frost numbers were lower than those of the nomadic interactions $(3<\mathrm{Fr}<500)$. Pursuit-type consumers thus spent relatively more time searching for patches, perhaps because most of their resource patches moved actively, in contrast to the stationary patches in the nomadic and semi-nomadic interactions. The pursuit-type Strathmann and Lessard numbers were also uniformly $<1$, meaning that patches were relatively shorter-lived and usually disappeared on their own before being fully exploited. Whereas the nomadic and semi-nomadic consumers could theoretically reproduce within patches, and would be forced to move only after depleting their resources, the pursuit-type consumers could never reproduce within patches. Instead, they relocated due to the patches' inherent ephemerality.

Pursuit-type interactions exclusively involved carnivorous species preying on smaller-bodied animals. With the exception of chimney swifts - aeroplankton, all interactions took place in aquatic environments, and, except for red knots feeding on clams and cockles, all prey were mobile and suspended in a fluid medium. The separation of the pursuit-type and (semi-) nomadic groups highlights the dynamical differences between consumer-resource interactions in fluid environments and those in benthic or terrestrial environments where prey are attached to surfaces (Strathmann 1990; Steele 1991; Carr et al. 2003). For instance, the marine tuna herring and terrestrial chimney swift - aeroplankton interactions were more similar to each other than either was to sea stars (a non-pelagic marine predator) or wolves (a non-aerial terrestrial predator). It is ironic that tuna, because of their voracity and pack hunting, have often been called the "wolves of the sea"-when in fact, they are better compared to an insectivorous bird, and wolves pursuing caribou are closer (at least in terms of patch dynamics) to crabs feeding on cockles (Figure 5).

The fourth cluster included 7 interactions, representing a varied collection of consumers, resources, and environments: microscopic and macroscopic; marine and terrestrial; carnivorous, herbivorous, and detritivorous. Their commonality was having all three dimensionless numbers close to the critical value of one-i.e., the timescales at which consumers moved between, reproduced within, and depleted patches were all similar to the patches' durations.

Consequently, these interactions were unlikely to satisfy any version of the mean-field assumption. Because movement, reproduction, and consumption processes were all marginal in these interactions, we called them triple-marginal.

For these consumers, small differences in reproductive or movement efficiency would mean the difference between effective and unsuccessful exploitation of patches. For instance, assuming diffusive movement, five of the consumers in this group would not be able to locate their resource patches, and based on the numbers we found, two consumers would be unable to locate patches even with directed movement. Several of the triple-marginal interactions did have large differences between the directed and diffusive Frost numbers, so if these consumers moved in a random-walk rather than directed fashion (as assumed for clustering), a spatial mean-field assumption might be justifiable. The triple-marginal cluster included four of the five 
planktonic consumers in our dataset (Bosmina, Copepods, Ctenophores, and Pseudoalteromonas), suggesting a hypothesis that planktonic consumers across a range of sizes may face similar challenges in gaining and maintaining access to resource patches. However, this cluster also included several non-planktonic interactions, including benthic and terrestrial predator-prey pairs and one terrestrial grazer.

The fifth cluster included only two interactions (fruit flies - marula trees and Vibrio - nutrients). Both had $\mathrm{Fr}>1, \operatorname{Str} \sim 1$, and Le $<<1$, indicating that the consumers could move between patches and possibly reproduce within them, but would not contribute significantly to resource depletion. Because these interactions involve consumers exploiting resources which appear suddenly and are more abundant than they can exploit, and because the fruit flies are literally consuming fallen fruit, we named them windfall interactions. These interactions were similar to the pursuit interactions in that movement between patches was possible and depletion was not $(\mathrm{Fr}>1$, Le $<<1)$. However, the windfall interactions had Str $\sim 1$, indicating that one generation of offspring could be produced within a nutrient plume or a fall of marula fruit before it diffused away or rotted, respectively. In these cases resource patches were both rich and moderately ephemeral, with a duration on the same order of magnitude as its consumer's generation time. The small size of this cluster compared to the others is notable. While our selection of interactions cannot be assumed to be representative, the relative frequency of different types of consumer-resource interactions would be an interesting direction for future research.

\section{Are bacteria really gazelles?}

Conceptualizing patchy consumer-resource dynamics in terms of dimensionless ratios can unite disparate ecological interactions into a common framework, and identify hidden similarities between them. However, these comparisons can at first appear quite abstract. To help make this style of thinking more concrete, we present a case study on one of the most surprising dynamic similarities we found, and the one that gives this paper its title: the similarity between Interaction 2, bacteria exploiting dissolved nutrients, and Interaction 26, gazelles grazing on the Mongolian steppe (Table 3, Figure 6). 
(a)

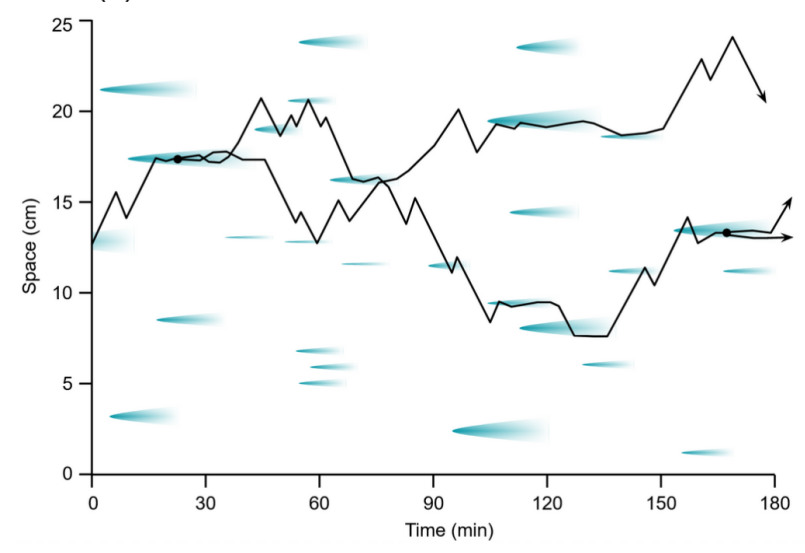

(b)

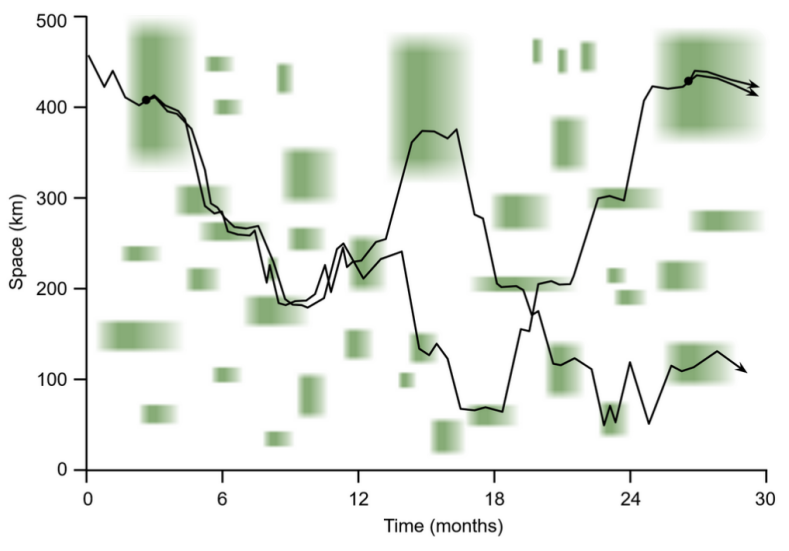

Figure 6. Patch exploitation by the marine bacterium Pseudoalteromonas haloplanktis and the Mongolian gazelle Procapra gutturosa are dynamically similar, despite differences in temporal scale on the order of $10^{3}$ and differences in spatial scale on the order of $10^{6}$. (a) Conceptual space-time diagram showing several bacteria (black lines) foraging for nutrient plumes from sinking phytoplankton cells (blue patches) in a simplified one-dimensional seascape. Black dots mark cell divisions, and hence the birth of new bacteria. (b) Similar diagram showing hypothetical paths of several gazelles browsing on patches of steppe vegetation which bloom following localized rains. As in a), black dots mark births of new organisms. The spatial and temporal scales of the patches are approximately accurate for each system, as are the speeds and generation times of the organisms. Note that for simplicity only a few trajectories are plotted; in reality the density of both organisms within their patches would be significantly higher.

Pseudoalteromonas haloplanktis is a cold-water planktonic bacterium which, in this interaction, seeks out a plume of nutrients left behind by a dead, sinking phytoplankton cell. Such plumes are typically separated by several $\mathrm{cm}$, and disperse via diffusion over about 15 minutes. Laboratory tracking has shown that $P$. haloplanktis can swim incredibly fast for its body size, moving up nutrient gradients at speeds of up to $0.4 \mathrm{~mm} / \mathrm{s}$ (Barbara \& Mitchell 2003), implying a directed Frost number of 2.0. Their doubling time of $\sim 3$ hours (Piette et al. 2010) is fast by human standards, but 12 times longer than the duration of an individual nutrient plume (implying Str $=0.083$ ), meaning that a bacterium dividing in a plume cannot expect its daughter cells to inherit the resource patch. Still, the relatively high density of bacteria that can aggregate in such a nutrient plume means that they can absorb the nutrients at about the same rate as diffusion carries the nutrients away, leading to a Lessard number approximately equal to one.

The Mongolian gazelle, Procapra gutturosa, differs in several respects from $P$. haloplanktis: to name a few, it is terrestrial, reproduces sexually, and possesses a body made of multiple eukaryotic cells that is 2.8 quadrillion times the mass of $P$. haloplanktis. Nevertheless, the foraging dynamics of gazelle are quantitatively similar, with (directed) $\mathrm{Fr}=12.3$, Str $=0.04$, and $\mathrm{Le}=3.0$, all on the same order of magnitude as corresponding numbers for the bacterium. An examination of the gazelles' natural history provides an explanation for this similarity: Herds of gazelles travel nearly constantly across the steppe, tracking patches of productive pasture that appear following rains. These patches last on the order of 30 days, long enough for herds to 
locate them, but an order of magnitude shorter than the gazelles' 2-year generation time. Still, the size and density of the herds (281 gazelles $\mathrm{km}^{-2}$, Olson et al. 2009) mean that they actively graze down their pastures.

The bacterium and the gazelle's interactions were closer to each other in logarithmic Fr-Str-Le space than to any others considered here. Both consumers are fast for their size and highly mobile relative to their resources and would be expected to spend the majority of their time inside patches. While these patches are long-lasting enough to enable easy discovery, they disappear too quickly to host population growth, meaning that each consumer must visit 10-20 patches per generation. At typical consumer densities, each patch is a finite resource, being depleted faster than it would be in the absence of the consumers. Figure 6 shows the conceptual space-time arrangement of patches and consumer trajectories for both these interactions. The dynamic similarity between them is intuitively obvious when comparing the two panels: if one ignores the axis labels and stylized patch shapes, the distribution of patches and the consumers' space-time trajectories are difficult to tell apart.

Of course, this is a deliberately simplified picture of these interactions' dynamics. In many respects, bacteria are not gazelles. Nutrient plumes are small compared to the spaces separating them, while patches of steppe vegetation vary widely in size and connectivity (Mueller et al. 2008). The consumers' sensory capabilities and foraging behaviors also differ significantly. While Pseudoalteromonas can perform chemotaxis, gazelles use multiple senses, memory, and social information to locate food. Bacterial division occurs whenever cellular development and nutrient assimilation permit, while gazelles have a seasonal reproductive cycle. Finally, the bacteria move in a three-dimensional environment, whereas the gazelles are restricted to the (approximately) flat surface of the earth. Nevertheless, dimensional analysis suggests that some of these differences in biology will be less important than dynamic similarities between the arrangement of patches in space and time, and the ways consumers travel between and exploit them over their lives' courses. Outside this dimensionless scaling framework, such a surprising dynamic similarity would not be apparent.

\section{Significance, perspective, and future directions}

As is the case for most dimensionless ratios, Fr, Str, and Le are based on a simplified caricature of the real world, and do not capture all dynamical similarities (or differences) that can exist between systems (e.g., density dependence, functional responses, other species interactions, responses to environmental drivers; Rogers \& Munch 2020). Further, each set of numbers characterizes an interaction, not a taxon. The same consumer could have different values for Fr, Str, and Le when feeding on a different resource, or even the same resource in a different place or time. Just as the nondimensional numbers can identify dynamic similarities across species, they can also identify dynamic differences within a single species as it grows, changes behavior, or encounters different resource and environmental conditions. In reality, resources are heterogeneous at a range of spatiotemporal scales, and patches can be nested inside each other. It is thus important to think critically about which of these scales are relevant for the 
consumers and the ecological questions at hand when calculating these dimensionless ratios. Examining the sensitivity of Fr, Str, and Le to patch scales in hierarchical resource landscapes would be a worthwhile direction for future investigation. Ultimately, though, it is important to remember that these ratios are not intended to be more precise than an order of magnitude: they are tools for reasoning about the relative magnitudes of different rates, not precisely modelling dynamics.

As a general framework, we believe dimensionless ratios are widely useful. Although the ratios explored here were derived from specific details about individual consumers and resources, clear groupings emerged that reflected similarities in life history (herbivorous vs. predatory consumers, mobile vs. stationary resources) and environment ("pelagic" vs. "benthic" systems). These separations hint at fundamental tradeoffs long discussed in ecology and evolution (Hutchinson 1961; Menge \& Sutherland 1987; Strathmann 1990). The dimensionless ratio approach is similar to the use of functional traits within community ecology (e.g., McGill et al. 2006), where the diversity of organism traits are reduced to their functional similarities in a comparative framework. Dimensionless descriptions highlight how species interact with each other and their environment and provide an opportunity for ecologists studying dynamically similar systems to learn from each other. It may also suggest experimentally tractable systems that can be used as proxies for dynamically similar interactions that occur at experimentally intractable spatial and temporal scales.

While our selection of consumer-resource pairs was extensive, it was not random or representative. For instance, our author group has little expertise with insects, so they are underrepresented in this paper. Additionally, we did not consider plants or fungi as consumers, even though they move between generations via seed or spore dispersal and can actively seek resource patches by extending roots or mycorrhizae. Finally, we found it surprisingly difficult to locate values for basic rates (speeds, reproductive and consumption rates, patch sizes, densities, etc.) in the literature, even for well-studied species, and most values we did find were published in older papers. While funders, publishers, and researchers may not consider measuring and reporting basic natural history information as "high-impact" or career-advancing ecology, their value to future researchers is hard to overstate (Greene 2005).

Understanding when patchiness matters and when it may be ignored is a constant challenge to ecological modellers. The Frost, Strathmann, and Lessard numbers can serve as diagnostic tools to assist in model development by identifying when mean-field approaches will work for a given system. For theoreticians seeking to understand patchy consumer-resource dynamics (e.g., Hein \& Martin 2019), empirical information on the Fr-Str-Le space occupied by real organisms can aid in model formulation and placement of realistic parameter bounds. The broad utility of these values is analogous to the Reynolds number in fluid dynamics: it is not the only number one needs to design an airplane, or predict the weather, but neither is possible without it. To model and understand ecological patchiness, we ought to start from a common quantification. The Frost, Strathmann, and Lessard numbers, proposed by Grünbaum (2012) 
and quantified here, may provide such a starting point from which to develop a deeper, more general understanding of patch dynamics in ecology.

\section{Acknowledgements}

This paper grew out of discussions at the Ecological Dissertations in the Aquatic Sciences symposium (Eco-DAS) at the University of Hawaii at Manoa in October 2018 and was supported by funding from the National Science Foundation (OCE-1356192) and the Association for the Sciences of Limnology and Oceanography. We are grateful to Dr. Paul Kemp and Kristina Remple for their work organizing the symposium, and to all our Eco-DAS 2018 friends for the stimulating discussions and good times. MLS was supported by a grant from the Simons Foundation (Award ID 602015). Gemma Carroll, Stephen Katz, Peter Dudley, and Patrick Ressler provided early feedback which greatly improved the manuscript. Finally, we would like to thank Daniel Grünbaum for supplying the ideas and framework that inspired this effort.

\section{Author contributions}

SSU conceived the study. All authors discussed the study's design, reviewed literature, and curated data. ANC, SSU, and TLR analyzed the data. ANC and SSU drafted the manuscript with input from all authors. ANC, CCS, JSS, SSU, and TLR contributed to figures and tables. All authors revised and edited the manuscript. 


\section{References}

Arditi, R. \& Dacorogna, B. (1988). Optimal Foraging on Arbitrary Food Distributions and the Definition of Habitat Patches. Am. Nat., 131, 837-846.

Barbara, G.M. \& Mitchell, J.G. (2003). Bacterial tracking of motile algae. FEMS Microbiol. Ecol., 44, 79-87.

Benoit-Bird, K.J., Battaile, B.C., Heppell, S.A., Hoover, B., Irons, D., Jones, N., et al. (2013). Prey Patch Patterns Predict Habitat Use by Top Marine Predators with Diverse Foraging Strategies. PLOS ONE, 8, e53348.

Beverton, R.J.H. \& Holt, S.J. (1959). A Review of the Lifespans and Mortality Rates of Fish in Nature, and Their Relation to Growth and Other Physiological Characteristics. In: Ciba Foundation Symposium - The Lifespan of Animals (Colloquia on Ageing). John Wiley \& Sons, Ltd, pp. 142-180.

Carr, M.H., Neigel, J.E., Estes, J.A., Andelman, S., Warner, R.R. \& Largier, J.L. (2003). Comparing Marine and Terrestrial Ecosystems: Implications for the Design of Coastal Marine Reserves. Ecol. Appl., 13, 90-107.

Charnov, E.L. (1976). Optimal foraging, the marginal value theorem. Theor. Popul. Biol., 9, 129-136.

Charnov, E.L. (1990). On evolution of age of maturity and the adult lifespan. J. Evol. Biol., 3, 139-144.

Charnov, E.L., Berrigan, D., Beverton, R.J.H., Harvey, P.H., Partridge, L. \& Southwood, S.R. (1991). Dimensionless numbers and the assembly rules for life histories. Philos. Trans. R. Soc. Lond. B. Biol. Sci., 332, 41-48.

Chesson, P. (2000). General Theory of Competitive Coexistence in Spatially-Varying Environments. Theor. Popul. Biol., 58, 211-237.

Codling, E.A. \& Dumbrell, A.J. (2012). Mathematical and theoretical ecology: linking models with ecological processes. Interface Focus, 2, 144-149.

Davis, M.A., Thompson, K., Grime, J.P. \& Liebhold, A. (2005). Invasibility: The Local Mechanism Driving Community Assembly and Species Diversity. Ecography, 28, 696-704.

DeAngelis, D.L. \& Yurek, S. (2017). Spatially Explicit Modeling in Ecology: A Review. Ecosystems, 20, 284-300.

Durrett, R. \& Levin, S. (1994). The Importance of Being Discrete (and Spatial). Theor. Popul. Biol., 46, 363-394.

Fortin, M.-J. (1999). Spatial Statistics in Landscape Ecology. In: Landscape Ecological Analysis: Issues and Applications (eds. Klopatek, J.M. \& Gardner, R.H.). Springer, New York, NY, pp. 253-279.

Greene, H.W. (2005). Organisms in nature as a central focus for biology. Trends Ecol. Evol., 20, 23-27.

Grünbaum, D. (2012). The logic of ecological patchiness. Interface Focus, 2, 150-155.

Gu, Z., Eils, R. \& Schlesner, M. (2016). Complex heatmaps reveal patterns and correlations in multidimensional genomic data. Bioinformatics, 32, 2847-2849.

Hanski, I. (1998). Metapopulation dynamics. Nature, 396, 41-49.

Hein, A.M. \& Martin, B.T. (2019). Information limitation and the dynamics of coupled ecological systems. Nat. Ecol. Evol.

Hellweger, F.L. (2018). Heterotrophic substrate specificity in the aquatic environment: The role of microscale patchiness investigated using modelling. Environ. Microbiol., 20, 
3825-3835.

Horne, J.K. \& Schneider, D.C. (1994). Analysis of Scale-Dependent Processes with Dimensionless Ratios. Oikos, 70, 201-211.

Hutchinson, G.. E.. (1961). The Paradox of the Plankton Author. Am. Nat., 95, 137-145.

Kermack, W.O., McKendrick, A.G. \& Walker, G.T. (1927). A contribution to the mathematical theory of epidemics. Proc. R. Soc. Lond. Ser. Contain. Pap. Math. Phys. Character, 115, 700-721.

de Knegt, H.J., Hengeveld, G.M., van Langevelde, F., de Boer, W.F. \& Kirkman, K.P. (2007).

Patch density determines movement patterns and foraging efficiency of large herbivores. Behav. Ecol., 18, 1065-1072.

Lasker, R. (1975). Field criteria for survival of anchovy larvae: The relation between inshore chlorophyll maximum layers and successful first feeding. Fish. Bull., 73, 453-462.

Lawton, J.H. (1999). Are There General Laws in Ecology? Oikos, 84, 177-192.

Levin, S.A. (1994). Patchiness in marine and terrestrial systems: from individuals to populations. Philos. Trans. R. Soc. Lond., 343 B, 99-103.

McGill, B.J., Enquist, B.J., Weiher, E. \& Westoby, M. (2006). Rebuilding community ecology from functional traits. Trends Ecol. Evol., 21, 178-185.

Menge, B.A. \& Sutherland, J.P. (1987). Community Regulation: Variation in Disturbance, Competition, and Predation in Relation to Environmental Stress and Recruitment. Am. Nat., 130, 730-757.

Morozov, A. \& Poggiale, J.-C. (2012). From spatially explicit ecological models to mean-field dynamics: The state of the art and perspectives. Ecol. Complex., From spatially explicit population models to mean-field dynamics, 10, 1-11.

Mueller, T., Olson, K.A., Fuller, T.K., Schaller, G.B., Murray, M.G. \& Leimgruber, P. (2008). In search of forage: predicting dynamic habitats of Mongolian gazelles using satellite-based estimates of vegetation productivity. J. Appl. Ecol. , 45, 649-658.

Murray, J.D. (1989). Mathematical Biology. Biomathematics. Springer-Verlag, Berlin Heidelberg.

Olson, K.A., Mueller, T., Bolortsetseg, S., Leimgruber, P., Fagan, W.F. \& Fuller, T.K. (2009). A mega-herd of more than 200,000 Mongolian gazelles Procapra gutturosa: a consequence of habitat quality. Oryx, 43, 149-153.

Piette, F., D’Amico, S., Struvay, C., Mazzucchelli, G., Renaut, J., Tutino, M.L., et al. (2010). Proteomics of life at low temperatures: trigger factor is the primary chaperone in the Antarctic bacterium Pseudoalteromonas haloplanktis TAC125. Mol. Microbiol., 76, 120-132.

Priyadarshi, A., Smith, S.L., Mandal, S., Tanaka, M. \& Yamazaki, H. (2019). Micro-scale patchiness enhances trophic transfer efficiency and potential plankton biodiversity. Sci. Rep., 9, 17243.

Reynolds, A.P., Richards, G., de la Iglesia, B. \& Rayward-Smith, V.J. (2006). Clustering Rules: A Comparison of Partitioning and Hierarchical Clustering Algorithms. J. Math. Model. Algorithms, 5, 475-504.

Rogers, T.L. \& Munch, S.B. (2020). Hidden similarities in the dynamics of a weakly synchronous marine metapopulation. Proc. Natl. Acad. Sci., 117, 479-485.

Safina, C. \& Burger, J. (1985). Common Tern Foraging: Seasonal Trends in Prey Fish Densities and Competition with Bluefish. Ecology, 66, 1457-1463.

Schubert, E. \& Rousseeuw, P.J. (2019). Faster k-Medoids Clustering: Improving the PAM, CLARA, and CLARANS Algorithms. In: Similarity Search and Applications, Lecture Notes in Computer Science (eds. Amato, G., Gennaro, C., Oria, V. \& Radovanović, M.). Springer International Publishing, Cham, pp. 171-187. 
Steele, J.H. (1991). Marine ecosystem dynamics: Comparison of scales. Ecol. Res., 6, 175-183.

Stephens, D.W. \& Dunbar, S.R. (1993). Dimensional analysis in behavioral ecology. Behav. Ecol., 4, 172-183.

Stocker, R. (2012). Marine Microbes See a Sea of Gradients. Science, 338, 628-633.

Strathmann, R.R. (1990). Why Life Histories Evolve Differently in the Sea1. Am. Zool., 30, 197-207.

Thorndike, R.L. (1953). Who belongs in the family? Psychometrika, 18, 267-276.

Urmy, S. \& Warren, J. (2018). Foraging hotspots of common and roseate terns: the influence of tidal currents, bathymetry, and prey density. Mar. Ecol. Prog. Ser., 590, 227-245.

Urmy, S.S. (2021). Visual trail following in colonial seabirds: theory, simulation, and remote observations. Ecol. Monogr., 91, 1-20.

Weimerskirch, H., Gault, A. \& Cherel, Y. (2005). Prey Distribution and Patchiness: Factors in Foraging Success and Efficiency of Wandering Albatrosses. Ecology, 86, 2611-2622.

Wiens, J.A. (1976). Population Responses to Patchy Environments. Annu. Rev. Ecol. Syst., 7, 81-120.

Yawata, Y., Carrara, F., Menolascina, F. \& Stocker, R. (2020). Constrained optimal foraging by marine bacterioplankton on particulate organic matter. Proc. Natl. Acad. Sci., 117, 25571-25579. 


\section{Supplementary material}

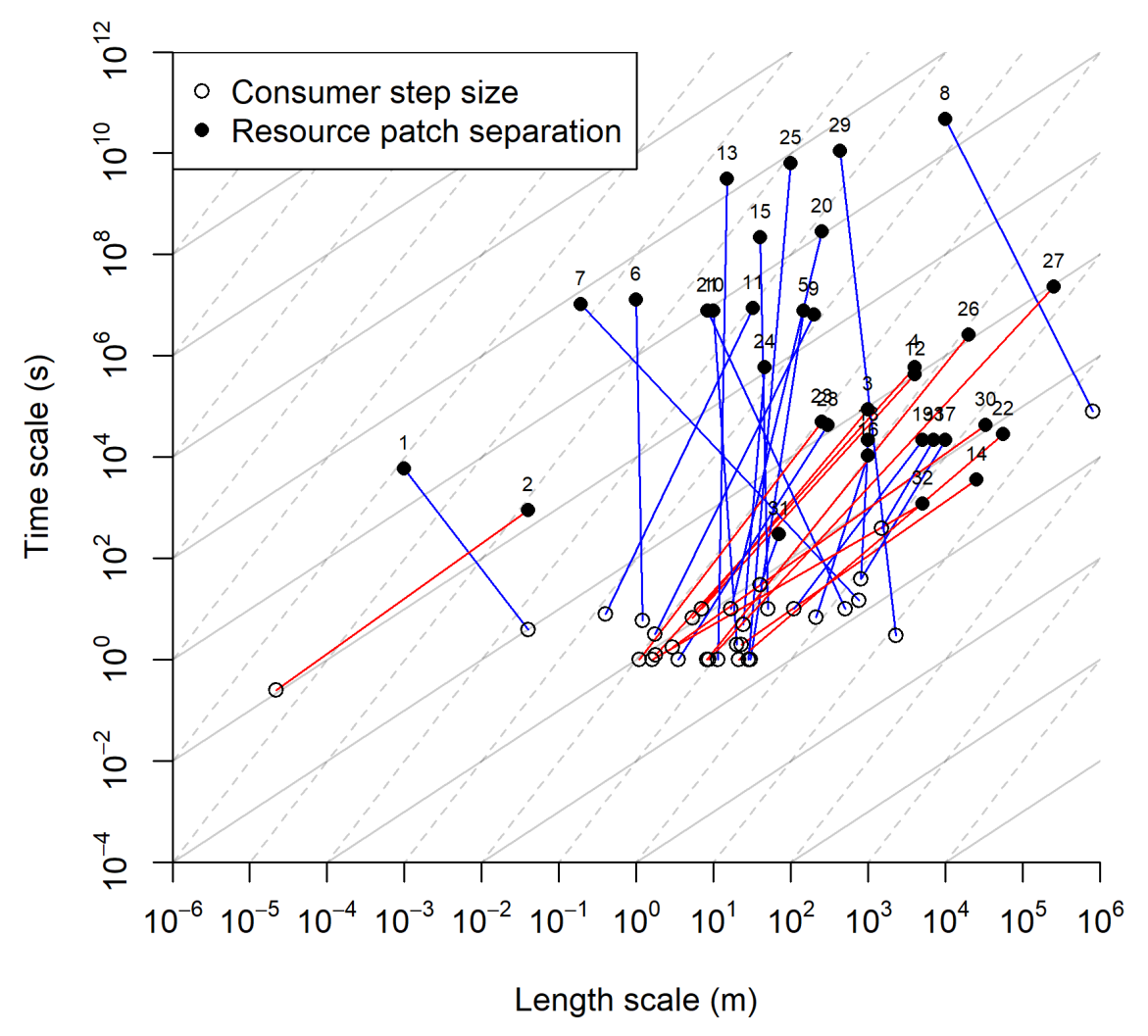

Figure S1. Patch availability by diffusive versus active movement. Critical Frost numbers for diffusive and directed searches, based on Grünbaum's (2012) Figure 1a. Filled points show the duration and separation scales of a resource patch. Each of these patch points is connected to an open point showing the "minimum step" of its consumer, placed at the turning interval $\tau$ and the distance traveled between turns at its representative velocity $s$ (Table 3 ). Numbered labels correspond to the consumer-resource interactions listed in Table 3 and all figures in the main text. A consumer can only find patches if its $\mathrm{Fr} \gtrsim 1$. On logarithmic length-time axes, the critical Frost numbers for directed and diffusive motion correspond to lines with slopes of 1 or 2 , respectively. The grid of solid and dotted lines in the background shows these slopes for reference. If the slope of the segment connecting a consumer to its resource is less than 1 , the resource is unavailable. If the slope is between 1 and 2 (red lines), patches can be located by a directed search, but not a random search. If the slope is $>2$ (blue lines), patches are available with any search strategy. 
bioRxiv preprint doi: https://doi.org/10.1101/2021.10.15.464607; this version posted October 20, 2021. The copyright holder for this preprint (which was not certified by peer review) is the author/funder, who has granted bioRxiv a license to display the preprint in perpetuity. It is made available under aCC-BY-NC-ND 4.0 International license.

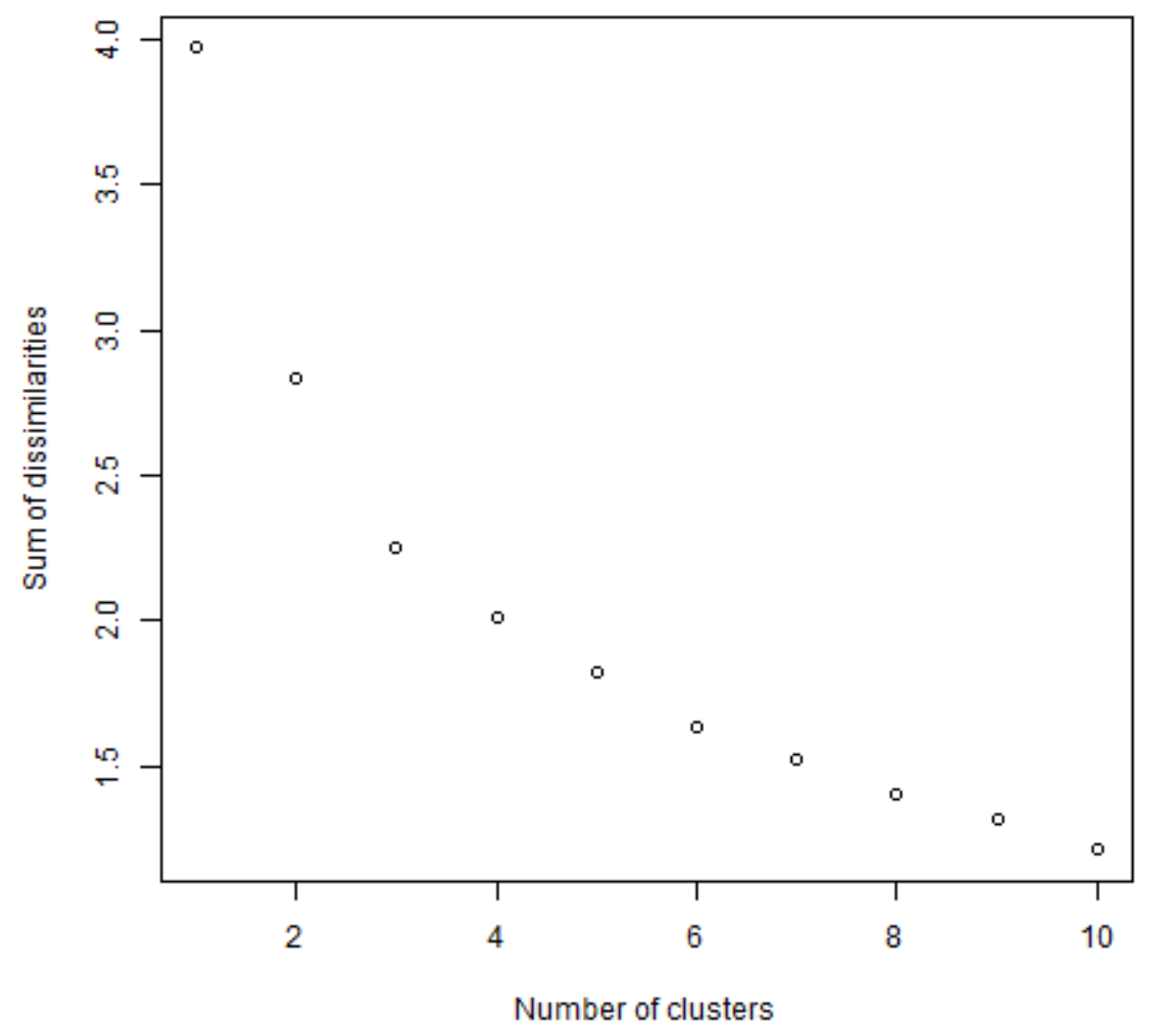

Figure S2. Elbow plot used to select the number of clusters. 
Table S1. Complete presentation of rates and dimensionless ratios for all consumer-resource pairs. Parameters are defined in Table 2. An abbreviated version of these data is given in Table 3. Consumer and resource quantities are presented in their original units from the primary literature, which vary from interaction to interaction. For instance, the "currency" of an interaction could be expressed in individuals or biomass, densities could be areal or volumetric, etc. However, when plugged into the equations in Table 2, the quantities within each row are dimensionally consistent. Each quantity is listed with its source in the literature, as both a URL permalink/DOU and author-year citation (cf. references below). Where the value of the quantity was not simply reported in the source paper, requiring some interpretation or calculations on our part, these are summarized in the “_comment” column. 


\section{Table S1 References}

Aguado-Giménez, F. \& García-García, B. (2005). Growth, food intake and feed conversion rates in captive Atlantic bluefin tuna (Thunnus thynnus Linnaeus, 1758) under fattening conditions. Aquaculture Research, 36, 610-614.

Alaska Department of Fish and Game. (2021). Caribou Species Profile. Alaska Department of Fish and Game. Available at: http://www.adfg.alaska.gov/index.cfm?adfg=caribou.main. Last accessed 29 July 2021.

Alexander, R. \& Imberger, J. (2009). Spatial distribution of motile phytoplankton in a stratified reservoir: the physical controls on patch formation. Journal of Plankton Research, 31, 101-118.

Bailey, H., Mate, B.R., Palacios, D.M., Irvine, L., Bograd, S.J. \& Costa, D.P. (2009). Behavioural estimation of blue whale movements in the Northeast Pacific from state-space model analysis of satellite tracks. Endangered Species Research, 10, 93-106.

Baker, R., Wilke, F. \& Baltzo, C. (1970). The Northern Fur Seal ( No. 360). U.S. Bureau of Commercial Fisheries, Washington, D.C.

Ballard, W.B., Ayres, L.A., Krausman, P.R., Reed, D.J. \& Fancy, S.G. (1997). Ecology of Wolves in Relation to a Migratory Caribou Herd in Northwest Alaska. Wildlife Monographs, 3-47.

Båmstedt, U. \& Martinussen, M.B. (2015). Ecology and behavior of Bolinopsis infundibulum (Ctenophora; Lobata) in the Northeast Atlantic. Hydrobiologia, 759, 3-14.

Barbara, G.M. \& Mitchell, J.G. (2003). Bacterial tracking of motile algae. FEMS Microbiology Ecology, 44, 79-87.

Beche, L.A. \& Resh, V.H. (2005). Preliminary studies on the life history of a wood-boring caddisfly, Heteroplectron californicum (Trichoptera: Calamoceratidae) in a coastal California stream. In: Proceedings of the 11th International Symposium on Trichoptera (2003, Osaka). Tokai University Press, pp. 39-43.

Benoit-Bird, K., Battaile, B., Nordstrom, C. \& Trites, A. (2013). Foraging behavior of northern fur seals closely matches the hierarchical patch scales of prey. Marine Ecology Progress Series, 479, 283-302.

Benvenuti, S., Bonadonna, F., Dall'Antonia, L. \& Gudmundsson, G.A. (1998). Foraging Flights of Breeding Thick-Billed Murres (Uria Lomvia) as Revealed by Bird-Borne Direction Recorders. The Auk, 115, 57-66. 
Bergey, E.A. \& Resh, V.H. (2006). Differential response of algae on small streambed substrates to floods. The American Midland Naturalist, 155, 270-277.

Berrill, M. (1982). The life cycle of the green crab Carcinus maenas at the Northern end of its range. Journal of Crustacean Biology, 2, 31-39.

Biber, P. (2021). Sea oats Uniola paniculata propagation guide. CPR Planting Guides. University of Southern Mississippi, Center for Plant Restoration and Coastal Plant Research, Ocean Springs, MS.

Bijleveld, A.I., MacCurdy, R.B., Chan, Y.-C., Penning, E., Gabrielson, R.M., Cluderay, J., et al. (2016). Understanding spatial distributions: negative density-dependence in prey causes predators to trade-off prey quantity with quality. Proceedings of the Royal Society $B$ : Biological Sciences, 283, 20151557.

Boeckmann, C. (2021). Growing kale: Planting, growing, and harvesting kale. The Old Farmer's Almanac. Available at: https://www.almanac.com/plant/kale. Last accessed 20 August 2021.

Boeuf, B.J.L., Naito, Y., Asaga, T., Crocker, D. \& Costa, D.P. (1992). Swim speed in a female northern elephant seal: metabolic and foraging implications. Can. J. Zool., 70, 786-795.

Bouma, H., Duiker, J.M.C., de Vries, P.P., Herman, P.M.J. \& Wolff, W.J. (2001). Spatial pattern of early recruitment of Macoma balthica (L.) and Cerastoderma edule (L.) in relation to sediment dynamics on a highly dynamic intertidal sandflat. Journal of Sea Research, 45, 79-93.

Boyle, S. \& Owens, S. (2012). North American beaver (Castor canadensis): a technical conservation assessment (USDA Forest Service, Rocky Mountain Region.). USDA Forest Service, Rocky Mountain Region.

Braly, K.S. (2009). Environmental variables influencing sexual reproduction of sea oats, Uniola paniculata (Poaceae) across a natural dune field on Bear Island, North Carolina. Master's Thesis. University of North Carolina, Wilmington.

Brenner, F.J. (1967). Spatial and Energy Requirements of Beavers. The Ohio Journal of Science, 67, 242-246.

Brodeur, R.D. \& Wilson, M.T. (1996). A review of the distribution, ecology and population dynamics of age-0 walleye pollock in the Gulf of Alaska. Fisheries Oceanography, 5, 148-166.

Broschart, M.R., Johnston, C.A. \& Naiman, R.J. (1989). Predicting beaver colony density in boreal landscapes. The Journal of Wildlife Management, 53, 929. 
Bruce, B.D. (2008). The biology and ecology of the white shark, Carcharodon carcharias. In: Sharks of the Open Ocean (eds. Camhi, M.D., Pikitch, E.K. \& Babcock, E.A.). Blackwell Publishing Ltd., Oxford, UK, pp. 69-81.

Bruce, B.D., Stevens, J.D. \& Malcolm, H. (2006). Movements and swimming behaviour of white sharks (Carcharodon carcharias) in Australian waters. Mar Biol, 150, 161-172.

Brumley, D.R., Carrara, F., Hein, A.M., Yawata, Y., Levin, S.A. \& Stocker, R. (2019). Bacteria push the limits of chemotactic precision to navigate dynamic chemical gradients. Proceedings of the National Academy of Sciences of the United States of America, 166, 10792-10797.

Buchwalter, D.B., Jenkins, J.J. \& Curtis, L.R. (2003). Temperature influences on water permeability and chlorpyrifos uptake in aquatic insects with differing respiratory strategies. Environmental Toxicology and Chemistry, 22, 2806-2812.

Bull, E.L. \& Beckwith, R.C. (1993). Diet and foraging behavior of Vaux's swifts in Northeastern Oregon. The Condor, 95, 1016-1023.

Burrows, M.T., Kawai, K. \& Hughes, R.N. (1999). Foraging by mobile predators on a rocky shore: underwater TV observations of movements of blennies Lipophrys pholis and crabs Carcinus maenas. Marine Ecology Progress Series, 187, 237-250.

Busher, P.E., Warner, R.J. \& Jenkins, S.H. (1983). Population density, colony composition, and local movements in two Sierra Nevadan beaver populations. Journal of Mammalogy, 64, 314-318.

Candy, J.R. \& Quinn, T.P. (1999). Behavior of adult chinook salmon (Oncorhynchus tshawytscha) in British Columbia coastal waters determined from ultrasonic telemetry. Canadian Journal of Zoology, 77, 1161-1169.

Carroll, G., Cox, M., Harcourt, R., Pitcher, B.J., Slip, D. \& Jonsen, I. (2017). Hierarchical influences of prey distribution on patterns of prey capture by a marine predator. Functional Ecology, 31, 1750-1760.

Chapman, C. (1988). Patch use and patch depletion by the spider and howling monkeys of Santa Rosa National Park, Costa Rica. Behaviour, 105, 99-116.

Chilson, P.B., Frick, W.F., Stepanian, P.M., Shipley, J.R., Kunz, T.H. \& Kelly, J.F. (2012). Estimating animal densities in the aerosphere using weather radar: To $Z$ or not to $Z$ ? Ecosphere, 3, art72. 
Chown, S.L. \& Klok, J. (2011). The ecological implications of physiological diversity in dung beetles. In: Ecology and evolution of dung beetles (eds. Simmons, L.W. \& Ridsdill-Smith, T.J.). John Wiley \& Sons, Ltd, Chichester, UK, pp. 200-219.

Cole, S.T., Wortham, C., Kunze, E. \& Owens, W.B. (2015). Eddy stirring and horizontal diffusivity from Argo float observations: Geographic and depth variability. Geophysical Research Letters, 42, 3989-3997.

Collins, W. (1979). Feeding behavior and habitat selection of deer and elk on northern Utah summer range. PhD Dissertation. Utah State University, Logan, UT.

Cornell Lab of Ornithology. (2017). Chimney swift identification. All About Birds. Available at: https://www.allaboutbirds.org/guide/Chimney Swift/id. Last accessed 28 March 2019.

Cortés, E. (2002). Incorporating Uncertainty into Demographic Modeling: Application to Shark Populations and Their Conservation. Conservation Biology, 16, 1048-1062.

Cressman, K. (1996). Current methods of desert locust forecasting at FAO 1. EPPO Bulletin, 26, $577-585$.

Croll, D.A., Gaston, A.J., Burger, A.E. \& Konnoff, D. (1992). Foraging behavior and physiological adaptation for diving in thick-billed murres. Ecology, 73, 344-356.

Croll, D.A., Gaston, A.J. \& Noble, D.G. (1991). Adaptive loss of mass in thick-billed murres. The Condor, 93, 496-502.

Dacke, M., Baird, E., Byrne, M., Scholtz, C.H. \& Warrant, E.J. (2013). Dung Beetles Use the Milky Way for Orientation. Current Biology, 23, 298-300.

Dann, P. \& Cullen, J.M. (1990). Survival, Patterns of Reproduction, and Lifetime Reproductive Output in Little Blue Penguins (Eudyptula minor) on Phillip Island, Victoria, Australia. In: Penguin Biology (eds. Davis, L.S. \& Darby, J.T.). Academic Press, San Diego, pp. 63-84.

Dann, P. \& Norman, F.I. (2006). Population regulation in Little Penguins (Eudyptula minor): the role of intraspecific competition for nesting sites and food during breeding. Emu - Austral Ornithology, 106, 289-296.

David, C.T. (1978). The relationship between body angle and flight speed in free-flying Drosophila. Physiological Entomology, 3, 191-195.

Day, R.J. (1972). Stand structure, succession, and use of southern Alberta's Rocky Mountain forest. Ecology, 53, 472-478.

De Vos, A., Justin O'Riain, M., Meyer, M.A., Kotze, P.G.H. \& Kock, A.A. (2015). Behavior of Cape fur seals (Arctocephalus pusillus pusillus) in relation to temporal variation in predation 
risk by white sharks (Carcharodon carcharias) around a seal rookery in False Bay, South Africa. Marine Mammal Science, 31, 1118-1131.

Dedual, M. (2005). Hidden secrets of Taupo trout revealed. Target Taupo: A newsletter for Taupo Anglers, 50.

DeRuiter, S.L., Langrock, R., Skirbutas, T., Goldbogen, J.A., Calambokidis, J., Friedlaender, A.S., et al. (2017). A multivariate mixed hidden Markov model for blue whale behaviour and responses to sound exposure. The Annals of Applied Statistics, 11, 362-392.

Deshpande, S.A., Carvalho, G.B., Amador, A., Phillips, A.M., Hoxha, S., Lizotte, K.J., et al. (2014). Quantifying Drosophila food intake: comparative analysis of current methodology. Nature Methods, 11, 535-540.

Dexter, R.W. (1969). Banding and nesting studies of the chimney swift, 1944-1968. The Ohio Journal of Science, 69, 193-213.

DiCostanzo, J. (1980). Population dynamics of a common tern colony. Journal of Field Ornithology, 51, 229-243.

Djawdan, M. \& Garland, T., Jr. (1988). Maximal Running Speeds of Bipedal and Quadrupedal Rodents. Journal of Mammalogy, 69, 765-772.

Donkor, N.T. \& Fryxell, J.M. (1999). Impact of beaver foraging on structure of lowland boreal forests of Algonquin Provincial Park, Ontario. Forest Ecology and Management, 118, 83-92.

Durbin, E.G., Durbin, A.G., S-mayda, T.J. \& Verity, P.G. (1983). Food limitation of production by adult Acartia tonsa in Narragansett Bay, Rhode Island1. Limnology and Oceanography, 28, 1199-1213.

Elliott, J.M. (1975). Number of meals in a day, maximum weight of food consumed in a day and maximum rate of feeding for brown trout, Salmo trutta L. Freshwater Biology, 5, 287-303.

Everett, R.L. \& Stevens, R. (1981). Deer mouse consumption of bitterbrush seed treated with four repellents. Journal of Range Management, 34, 393.

FAO. (2021). Rainbow Trout - Growth. Available at: http://www.fao.org/fishery/affris/species-profiles/rainbow-trout/growth/en/. Last accessed 23 July 2021.

FAO Migratory Pests Group. (2004). Desert Locust Information Service of FAO: Locust FAQs. Available at: http://www.fao.org/ag/locusts/oldsite/LOCFAQ.htm. Last accessed 23 July 2021. 
Farrell, A.P. (2008). Comparisons of swimming performance in rainbow trout using constant acceleration and critical swimming speed tests. Journal of Fish Biology, 72, 693-710.

Feder, H.M. (1970). Growth and predation by the ochre sea star, Pisaster ochraceus (Brandt), in Monterey Bay, California. Ophelia, 8, 161-185.

Fernandez-Gimenez, M.E. \& Allen-Diaz, B. (1999). Testing a non-equilibrium model of rangeland vegetation dynamics in Mongolia. Journal of Applied Ecology, 36, 871-885.

Folger, P. (2013). Severe thunderstorms and tornadoes in the United States (CRS Report for Congress No. R40097). Congressional Research Service.

Ford, J.K.B. (2009). Killer Whale. In: Encyclopedia of marine mammals. Academic Press, Burlington,MA, pp. 650-657.

Ford, J.K.B. \& Ellis, G.M. (2006). Selective foraging by fish-eating killer whales Orcinus orca in British Columbia. Marine Ecology Progress Series, 316, 185-199.

Ford, J.K.B., Wright, B.M., Ellis, G.M. \& Candy, J.R. (2009). Chinook salmon predation by resident killer whales: seasonal and regional selectivity, stock identity of prey, and consumption rates ( No. 2010). DFO Canada, Canadian Science Advisory Secretariat.

Ford, S.M. \& Davis, L.C. (1992). Systematics and body size: Implications for feeding adaptations in new world monkeys. American Journal of Physical Anthropology, 88, 415-468.

Frair, J.L., Merrill, E.H., Visscher, D.R., Fortin, D., Beyer, H.L. \& Morales, J.M. (2005). Scales of movement by elk (Cervus elaphus) in response to heterogeneity in forage resources and predation risk. Landscape Ecology, 20, 273-287.

Frank, D. (1992). Body mass and nest reliefs in Common Terns Sterna hirundo exposed to different feeding conditions. Ardea, 80, 57.

Fryxell, J.M. \& Doucet, C.M. (1993). Diet choice and the functional response of beavers. Ecology, 74, 1297-1306.

Fuller, T., Mech, L. \& Cochrane, J. (2003). Wolf Population Dynamics. In: Wolves: Behavior, Ecology, and Conservation (eds. Mech, L.D. \& Boitani, L.). University of Chicago Press, Chicago, pp. 161-191.

Gadd, M.E. (2002). The impact of elephants on the marula tree Sclerocarya birrea. African Journal of Ecology, 40, 328-336.

Gales, R. \& Green, B. (1990). The annual energetics cycle of little penguins (Eudyptula minor). Ecology, 71, 2297-2312. 
Gaston, A.J., de Forest, L.N., Donaldson, G. \& Noble, D.G. (1994). Population parameters of thick-billed murres at coats island, Northwest Territories, Canada. The Condor, 96, 935-948.

Gauthier, G., Gauthier, M. \& Christen, R. (1995). Phylogenetic Analysis of the Genera Alteromonas, Shewanella, and Moritella Using Genes Coding for Small-Subunit rRNA Sequences and Division of the Genus Alteromonas into Two Genera, Alteromonas (Emended) and Pseudoalteromonas gen. nov., and Proposal of Twelve New Species Combinations. International Journal of Systematic and Evolutionary Microbiology, 45, 755-761.

Gaymer, C.F. \& Himmelman, J.H. (2002). Mussel beds in deeper water provide an unusual situation for competitive interactions between the seastars Leptasterias polaris and Asterias vulgaris. Journal of Experimental Marine Biology and Ecology, 277, 13-24.

Gaymer, C.F., Himmelman, J.H. \& Johnson, L.E. (2002). Use of prey resources by the seastars Leptasterias polaris and Asterias vulgaris: a comparison between field observations and laboratory experiments. Journal of Experimental Marine Biology and Ecology, 262, 13-30.

Gilpatrick, J.W. \& Perryman, W.L. (2008). Geographic variation in external morphology of North Pacific and Southern Hemisphere blue whales (Balaenoptera musculus). Journal of Cetacean Research and Management, 10, 9-21.

Goldbogen, J.A., Calambokidis, J., Oleson, E., Potvin, J., Pyenson, N.D., Schorr, G., et al. (2011). Mechanics, hydrodynamics and energetics of blue whale lunge feeding: efficiency dependence on krill density. Journal of Experimental Biology, 214, 131-146.

Gómez-Gutiérrez, J., Feinberg, L.R., Shaw, T. \& Peterson, W.T. (2006). Variability in brood size and female length of Euphausia pacifica among three populations in the North Pacific. Marine Ecology Progress Series, 323, 185-194.

Gong, Z., Andrews, M., Jagannathan, S., Patel, R., Jech, J.M., Makris, N.C., et al. (2010). Low-frequency target strength and abundance of shoaling Atlantic herring (Clupea harengus) in the Gulf of Maine during the Ocean Acoustic Waveguide Remote Sensing 2006 Experiment. The Journal of the Acoustical Society of America, 127, 104-123.

Green, H.V. (1936). The beaver of the Riding Mountain, Manitoba: an ecological study and commentary. The Canadian Field-Naturalist, 50, 1-8, 21-23, 36-50, 1-67, 85-92.

Greer, A.T., Cowen, R.K., Guigand, C.M., McManus, M.A., Sevadjian, J.C. \& Timmerman, A.H.V. (2013). Relationships between phytoplankton thin layers and the fine-scale vertical 
distributions of two trophic levels of zooplankton. Journal of Plankton Research, 35, 939-956.

Gunn, J.S., Clear, N.P., Carter, T.I., Rees, A.J., Stanley, C.A., Farley, J.H., et al. (2008). Age and growth in southern bluefin tuna, Thunnus maccoyii (Castelnau): Direct estimation from otoliths, scales and vertebrae. Fisheries Research, 92, 207-220.

Hall, J.B. (2002). Sclerocarya birrea (PROTA) - PlantUse English. PROTA (Plant Resources of Tropical Africa/ Ressources végétales de l'Afrique tropicale). Available at: https://uses.plantnet-project.org/en/Sclerocarya birrea (PROTA). Last accessed 17 August 2021.

Hanks, E.M., Hooten, M.B., Johnson, D.S. \& Sterling, J.T. (2011). Velocity-Based Movement Modeling for Individual and Population Level Inference. PLOS ONE, 6, e22795.

Hart, D.D. (1981). Foraging and resource patchiness: field experiments with a grazing stream insect. Oikos, 37, 46.

Hart, D.D. \& Resh, V.H. (1980). Movement patterns and foraging ecology of a stream caddisfly larva. Can. J. Zool., 58, 1174-1185.

Hebblewhite, M., White, C.A., Nietvelt, C.G., McKenzie, J.A., Hurd, T.E., Fryxell, J.M., et al. (2005). Human Activity Mediates a Trophic Cascade Caused by Wolves. Ecology, 86, 2135-2144.

Hedenström, A. \& Åkesson, S. (2017). Flight speed adjustment by three wader species in relation to winds and flock size. Animal Behaviour, 134, 209-215.

Himmelman, J. \& Dutil, C. (1991). Distribution, population structure and feeding of subtidal seastars in the northern Gulf of St. Lawrence. Marine Ecology Progress Series, 76, 61-72.

Hobbs, N.T. \& Swift, D.M. (1988). Grazing in herds: When are nutritional benefits realized? The American Naturalist, 131, 760-764.

Hofmeyr, G.J.G. (2015). Arctocephalus pusillus The IUCN red list of threatened species. International Union for Conservation of Nature. Available at: http://www.iucnredlist.org/details/2060/0. Last accessed 3 April 2019.

Holliday, D.V., Greenlaw, C.F. \& Donaghay, P.L. (2010). Acoustic scattering in the coastal ocean at Monterey Bay, CA, USA: Fine-scale vertical structures. Continental Shelf Research, 30, 81-103.

Holm, D.A., Ochsenwald, W.L. \& Owen, L. (1998). Arabian Desert. Encyclopedia Britannica. 
Horsburgh, J.M., Morrice, M., Lea, M. \& Hindell, M.A. (2008). Determining feeding events and prey encounter rates in a southern elephant seal: a method using swim speed and stomach temperature. Marine Mammal Science, 24, 207-217.

Huntley, M.E. \& Lopez, M.D.G. (1992). Temperature-dependent production of marine copepods: a global synthesis. The American Naturalist, 140, 201-242.

Innes, S., Lavigne, D.M., Earle, W.M. \& Kovacs, K.M. (1987). Feeding rates of seals and whales. The Journal of Animal Ecology, 56, 115.

Ito, T.Y., Miura, N., Lhagvasuren, B., Enkhbileg, D., Takatsuki, S., Tsunekawa, A., et al. (2006). Satellite tracking of Mongolian gazelles (Procapra gutturosa) and habitat shifts in their seasonal ranges. Journal of Zoology, 269, 291-298.

Ives, P.M. (1978). How discriminating are cabbage butterflies? Austral Ecology, 3, 261-276. James, M.R. (1987). Seasonal changes in metabolic rates of zooplankton and their importance to phytoplankton production in two New Zealand lakes of contrasting trophic state. New Zealand Journal of Marine and Freshwater Research, 21, 573-583.

Jeffrey, K.M., Côté, I.M., Irvine, J.R. \& Reynolds, J.D. (2017). Changes in body size of Canadian Pacific salmon over six decades. Canadian Journal of Fisheries and Aquatic Sciences, 74, 191-201.

Jensen, K.T. \& Jensen, J.N. (1985). The importance of some epibenthic predators on the density of juvenile benthic macrofauna in the Danish Wadden Sea. Journal of Experimental Marine Biology and Ecology, 89, 157-174.

Jiang, Z., Takatsuki, S., Gao, Z. \& Jin, K. (1998). The present status, ecology and conservation of the Mongolian gazelle, Procapra gutturosa : a review. Mammal Study, 23, 63-78.

Johnson, E.A., Miyanishi, K. \& Kleb, H. (1994). The Hazards of Interpretation of Static Age Structures as Shown by Stand Reconstructions in a Pinus Contorta -- Picea Engelmannii Forest. Journal of Ecology, 82, 923-931.

Johnson, I., Brinkman, T., Lake, B. \& Brown, C. (2017). Winter hunting behavior and habitat selection of wolves in a low-density prey system. Wildlife Biology, 2017.

Jones, R.E. (1977). Search behaviour: a study of three caterpillar species. Behaviour, 60, 237-259.

Kaltenberg, A. \& Benoit-Bird, K. (2009). Diel behavior of sardine and anchovy schools in the California Current System. Marine Ecology Progress Series, 394, 247-262. 
Kasuya, T., Ishimaru, T. \& Murano, M. (2000). Seasonal Variations in Abundance and Size Composition of the Lobate Ctenophore Bolinopsis Mikado (Moser) in Tokyo Bay, Central Japan. Journal of Oceanography, 56, 419-427.

Katano, I., Mitsuhashi, H., Isobe, Y., Sato, H. \& Oishi, T. (2007). Group size of feeding stream case-bearing caddisfly grazers and resource abundance. Basic and Applied Ecology, 8, 269-279.

Kenefic, L.S. \& Nyland, R.D. (1999). Sugar Maple Height-Diameter and Age-Diameter Relationships in an Uneven-Aged Northern Hardwood Stand. Northern Journal of Applied Forestry, 16, 43-47.

Kingsolver, J.G. (2000). Feeding, growth, and the thermal environment of cabbage white caterpillars, Pieris rapae. Physiological and Biochemical Zoology, 73, 621-628.

Klimley, A.P., Beavers, S.C., Curtis, T.H. \& Jorgensen, S.J. (2002). Movements and Swimming Behavior of Three Species of Sharks in La Jolla Canyon, California. Environmental Biology of Fishes, 63, 117-135.

Klimley, A.P., Le Boeuf, B.J., Cantara, K.M., Richert, J.E., Davis, S.F., Van Sommeran, S., et al. (2001). The hunting strategy of white sharks (Carcharodon carcharias) near a seal colony. Marine Biology, 138, 617-636.

Kock, A.A.A., Johnson, R.L., Bester, M.N., Compagno, L., Cliff, G., Dudley, S., et al. (2006). White Shark abundance: not a causative factor in numbers of shark bite incidents. In: Finding a balance: White shark conservation and recreational safety in the inshore waters of Cape Town, South Africa; proceedings of a specialist workshop. Presented at the WWF South Africa Report Series, pp. 1-20.

Koprowski, J.L. (1994). Sciurus carolinensis. Mammalian Species, 1-9.

Kraan, C., van der Meer, J., Dekinga, A. \& Piersma, T. (2009). Patchiness of macrobenthic invertebrates in homogenized intertidal habitats: hidden spatial structure at a landscape scale. Marine Ecology Progress Series, 383, 211-224.

Kryger, U., Cole, K.S., Tukker, R. \& Scholtz, C.H. (2006). Biology and ecology of Circellium bacchus (Fabricius 1781) (Coleoptera Scarabaeidae), a South African dung beetle of conservation concern. Tropical Zoology, 19, 185-207.

Kuhara, N., Nakano, S. \& Miyasaka, H. (2001). Alterations in the grazing activities of cased caddisfly larvae in response to variations in predation risk and resource level. Ecological Research, 16, 705-714. 
Lang, Z.C. (2006). Stipa baicalensis. Floras of China @ efloras.org. Available at: http://www.efloras.org/florataxon.aspx?flora id=2\&taxon id=200026367. Last accessed 17 August 2021.

Larter, N.C. \& Allaire, D.G. (2018). Dehcho boreal caribou study progress report (Technical Report). Environment and Natural Resources, Government of the Northwest Territories.

Levey, D.J. (1990). Habitat-dependent fruiting behaviour of an understorey tree, Miconia centrodesma, and tropical treefall gaps as keystone habitats for frugivores in Costa Rica. Journal of Tropical Ecology, 6, 409-420.

Lewis, A.R. (1980). Patch by gray squirrels and optimal foraging. Ecology, 61, 1371-1379.

Lutcavage, M.E., Brill, R.W., Skomal, G.B., Chase, B.C., Goldstein, J.L. \& Tutein, J. (2000). Tracking adult North Atlantic bluefin tuna (Thunnus thynnus) in the northwestern Atlantic using ultrasonic telemetry. Marine Biology, 137, 347-358.

Mansourian, S., Enjin, A., Jirle, E.V., Ramesh, V., Rehermann, G., Becher, P.G., et al. (2018). Wild African Drosophila melanogaster are seasonal specialists on marula fruit. Current Biology, 28, 3960-3968.

Markow, T.A. \& O’Grady, P. (2008). Reproductive ecology of Drosophila. Functional Ecology, 22, 747-759.

Massachusetts Division of Fisheries and Wildlife. (2021). Learn about squirrels. Mass.gov. Available at: https://www.mass.gov/service-details/learn-about-squirrels. Last accessed 26 July 2021.

Matonis, M.S., Walters, M.B. \& Millington, J.D.A. (2011). Gap-, stand-, and landscape-scale factors contribute to poor sugar maple regeneration after timber harvest. Forest Ecology and Management, 262, 286-298.

McClain, C.R. \& Hardy, S.M. (2010). The dynamics of biogeographic ranges in the deep sea. Proceedings of the Royal Society B: Biological Sciences, 277, 3533-3546.

McLean, K.A., Trainor, A.M., Asner, G.P., Crofoot, M.C., Hopkins, M.E., Campbell, C.J., et al. (2016). Movement patterns of three arboreal primates in a Neotropical moist forest explained by LiDAR-estimated canopy structure. Landscape Ecol, 31, 1849-1862.

Mech, L.D. (1994). Regular and Homeward Travel Speeds of Arctic Wolves. Journal of Mammalogy, 75, 741-742.

Mech, L.D. \& Boitani, L. (Eds.). (2003). Wolves: Behavior, Ecology, and Conservation. University of Chicago Press, Chicago. 
Menge, B.A., Berlow, E.L., Blanchette, C.A., Navarrete, S.A. \& Yamada, S.B. (1994). The keystone species concept: variation in interaction strength in a rocky intertidal habitat. Ecological Monographs, 64, 249-286.

Meroni, M., Rembold, F., Verstraete, M., Gommes, R., Schucknecht, A. \& Beye, G. (2014). Investigating the relationship between the inter-annual variability of satellite-derived vegetation phenology and a proxy of biomass production in the Sahel. Remote Sensing, 6 , 5868-5884.

Millar, J.S. \& Zammuto, R.M. (1983). Life histories of mammals: An analysis of life tables. Ecology, 64, 631-635.

Miller, C. (2000). Drosophila melanogaster. Animal Diversity Web. Available at: https://animaldiversity.org/accounts/Drosophila melanogaster/. Last accessed 26 July 2021.

Miller, T.E., Gornish, E.S. \& Buckley, H.L. (2010). Climate and coastal dune vegetation: disturbance, recovery, and succession. Plant Ecology, 206, 97-104.

Mills, K.J., Patterson, B.R. \& Murray, D.L. (2006). Effects of Variable Sampling Frequencies on GPS Transmitter Efficiency and Estimated Wolf Home Range Size and Movement Distance. Wildlife Society Bulletin, 34, 1463-1469.

Milton, K. (1981). Estimates of reproductive parameters for free-ranging Ateles geoffroyi. Primates, 22, 574-579.

Milton, K., Windsor, D.M., Morrison, D.W. \& Estribi, M.A. (1982). Fruiting phenologies of two Neotropical Ficus species. Ecology, 63, 752-762.

Moline, M.A., Benoit-Bird, K.J., Robbins, I.C., Schroth-Miller, M., Waluk, C.M. \& Zelenke, B. (2010). Integrated measurements of acoustical and optical thin layers II: Horizontal length scales. Continental Shelf Research, 30, 29-38.

Morrison, T.A., Chiyo, P.I., Moss, C.J. \& Alberts, S.C. (2005). Measures of dung bolus size for known-age African elephants (Loxodonta africana): implications for age estimation. Journal of Zoology, 266, 89-94.

Mubalama, M. \& Sikubwabo, C. (2002). Rate of decay of elephant dung in the central sector of Parc National des Virunga, Democratic Republic of Congo. Pachyderm, 33, 43-49.

Mueller, T., Olson, K.A., Dressler, G., Leimgruber, P., Fuller, T.K., Nicolson, C., et al. (2011). How landscape dynamics link individual- to population-level movement patterns: a multispecies comparison of ungulate relocation data: Population-level movement patterns. Global Ecology and Biogeography, 20, 683-694. 
Mueller, T., Olson, K.A., Fuller, T.K., Schaller, G.B., Murray, M.G. \& Leimgruber, P. (2008). In search of forage: predicting dynamic habitats of Mongolian gazelles using satellite-based estimates of vegetation productivity. Journal of Applied Ecology, 45, 649-658.

Nealis, V.G., Jones, R.E. \& Wellington, W.G. (1984). Temperature and development in host-parasite relationships. Oecologia, 61, 224-229.

Newlands, N.K., Lutcavage, M.E. \& Pitcher, T.J. (2004). Analysis of foraging movements of Atlantic bluefin tuna (Thunnus thynnus): individuals switch between two modes of search behaviour. Population Ecology, 46, 39-53.

Newlands, N.K. \& Porcelli, T.A. (2008). Measurement of the size, shape and structure of Atlantic bluefin tuna schools in the open ocean. Fisheries Research, 91, 42-55.

Niles, L.J., Sitters, H.P., Dey, A.D., Atkinson, P.W., Baker, A.J., Bennett, K.A., et al. (2008). Status of the red knot (Calidris canutus rufa) in the Western Hemisphere. Studies in avian biology. Cooper Ornithological Society, Camarillo, CA.

NOAA Fisheries. (2021). Northern Fur Seal. NOAA. Available at: https://www.fisheries.noaa.gov/species/northern-fur-seal. Last accessed 27 July 2021.

Nordstrom, C.A., Benoit-Bird, K.J., Battaile, B.C. \& Trites, A.W. (2013). Northern fur seals augment ship-derived ocean temperatures with higher temporal and spatial resolution data in the eastern Bering Sea. Deep Sea Research Part II: Topical Studies in Oceanography, 94, 257-273.

Olson, K.A., Fuller, T.K., Schaller, G.B., Lhagvasuren, B. \& Odonkhuu, D. (2006). Reproduction, neonatal weights, and first-year survival of Mongolian gazelles (Procapra gutturosa). Journal of Zoology, 265, 227-233.

Olson, K.A., Mueller, T., Bolortsetseg, S., Leimgruber, P., Fagan, W.F. \& Fuller, T.K. (2009). A mega-herd of more than 200,000 Mongolian gazelles Procapra gutturosa: a consequence of habitat quality. Oryx, 43, 149-153.

Oudman, T., Bijleveld, A.I., Kavelaars, M.M., Dekinga, A., Cluderay, J., Piersma, T., et al. (2016). Diet preferences as the cause of individual differences rather than the consequence. Journal of Animal Ecology, 85, 1378-1388.

Oudman, T., Piersma, T., Ahmedou Salem, M.V., Feis, M.E., Dekinga, A., Holthuijsen, S., et al. (2018). Resource landscapes explain contrasting patterns of aggregation and site fidelity by red knots at two wintering sites. Movement Ecology, 6, 24. 
Overholtz, W.J. (2002). The Gulf of Maine-Georges Bank Atlantic herring (Clupea harengus): spatial pattern analysis of the collapse and recovery of a large marine fish complex. Fisheries Research, 57, 237-254.

Overholtz, W.J., Jacobson, L.D., Melvin, G.D., Cieri, M., Power, M.E., Libby, D., et al. (2004). Stock assessment of the Gulf of Maine - Georges Bank Atlantic Herring Complex, 2003. Northeast Fisheries Science Center Reference Document 04-06. National Oceanic and Atmospheric Administration.

Paine, R.T. (1974). Intertidal community structure: Experimental studies on the relationship between a dominant competitor and its principal predator. Oecologia, 15, 93-120.

Penguin info. (2019). Information about locomotion and movement of penguins. Available at: http://www.pinguins.info/Engels/Voortdiepte eng.html. Last accessed 2 April 2019.

Peters, R.H. \& Downing, J.A. (1984). Empirical analysis of zooplankton filtering and feeding rates1. Limnology and Oceanography, 29, 763-784.

Piersma, T. (2012). What is habitat quality? Dissecting a research portfolio on shorebirds. In: Birds and habitat (ed. Fuller, R.J.). Cambridge University Press, Cambridge, pp. 383-407. Piette, F., D’Amico, S., Struvay, C., Mazzucchelli, G., Renaut, J., Tutino, M.L., et al. (2010). Proteomics of life at low temperatures: trigger factor is the primary chaperone in the Antarctic bacterium Pseudoalteromonas haloplanktis TAC125. Molecular Microbiology, 76, 120-132.

Pitcher, T.J. \& Partridge, B.L. (1979). Fish school density and volume. Marine Biology, 54, 383-394.

Plumptre, A.J. \& Harris, S. (1995). Estimating the biomass of large mammalian herbivores in a tropical montane forest: a method of faecal counting that avoids assuming a "steady state" system. The Journal of Applied Ecology, 32, 111.

Ramos-Fernández, G., Mateos, J.L., Miramontes, O., Cocho, G., Larralde, H. \& Ayala-Orozco, B. (2004). Lévy walk patterns in the foraging movements of spider monkeys (Ateles geoffroyi). Behav Ecol Sociobiol, 55, 223-230.

Reeves, R.R., Clapham, P.J., Brownell, R.L. \& Silber, G.K. (1998). Recovery plan for the blue whale (Balaenoptera musculus). National Marine Fisheries Service.

Reynolds, A.M. \& Frye, M.A. (2007). Free-Flight Odor Tracking in Drosophila Is Consistent with an Optimal Intermittent Scale-Free Search. PLOS ONE, 2, e354.

Richards, S.W. (1982). Aspects of the biology of Ammodytes americanus from the St. Lawrence River to Chesapeake Bay, 1972-75, including a comparison of the Long Island Sound 
postlarvae with Ammodytes dubius. Journal of Northwest Atlantic Fishery Science, 3, 93-104.

Rickbeil, G.J.M., Merkle, J.A., Anderson, G., Atwood, M.P., Beckmann, J.P., Cole, E.K., et al. (2019). Plasticity in elk migration timing is a response to changing environmental conditions. Global Change Biology, 25, 2368-2381.

Rines, J.E.B., McFarland, M.N., Donaghay, P.L. \& Sullivan, J.M. (2010). Thin layers and species-specific characterization of the phytoplankton community in Monterey Bay, California, USA. Continental Shelf Research, 30, 66-80.

Robles, C., Sherwood-Stephens, R. \& Alvarado, M. (1995). Responses of a key intertidal predator to varying recruitment of its prey. Ecology, 76, 565-579.

Robles, C.D., Desharnais, R.A., Garza, C., Donahue, M.J. \& Martinez, C.A. (2009). Complex equilibria in the maintenance of boundaries: experiments with mussel beds. Ecology, 90, 985-995.

Rockwood, R.C., Elliott, M.L., Saenz, B., Nur, N. \& Jahncke, J. (2020). Modeling predator and prey hotspots: Management implications of baleen whale co-occurrence with krill in Central California. PLOS ONE, 15, e0235603.

Rogers, T.L. \& Elliott, J.K. (2013). Differences in relative abundance and size structure of the sea stars Pisasterochraceus and Evasteriastroschelii among habitat types in Puget Sound, Washington, USA. Mar Biol, 160, 853-865.

Romine, J.G., Perry, R.W., Johnston, S.V., Fitzer, C.W., Pagliughi, S.W. \& Blake, A.R. (2014). Identifying when tagged fishes have been consumed by piscivorous predators: application of multivariate mixture models to movement parameters of telemetered fishes. Animal Biotelemetry, 2, 3.

Russell, R.W. (1999). Precipitation scrubbing of aerial plankton: inferences from bird behavior. Oecologia, 118, 381-387.

Ryan, J.P., McManus, M.A. \& Sullivan, J.M. (2010). Interacting physical, chemical and biological forcing of phytoplankton thin-layer variability in Monterey Bay, California. Continental Shelf Research, 30, 7-16.

Santora, J.A., Ralston, S. \& Sydeman, W.J. (2011). Spatial organization of krill and seabirds in the central California Current. ICES Journal of Marine Science, 68, 1391-1402.

Semmens, J.M., Payne, N.L., Huveneers, C., Sims, D.W. \& Bruce, B.D. (2013). Feeding requirements of white sharks may be higher than originally thought. Sci $\operatorname{Rep}, 3,1471$. 
Showler, A.T. (2015). The desert locust in Africa and Western Asia: complexities of war, politics, perilous terrain, and development. In: Radcliffe's IPM World Textbook (eds. Hutchinson, W.D. \& Cancelado, R.E.). University of Minnesota, St. Paul, MN.

Singh, G.G., Markel, R.W., Martone, R.G., Salomon, A.K., Harley, C.D.G. \& Chan, K.M.A. (2013). Sea otters homogenize mussel beds and reduce habitat provisioning in a rocky intertidal ecosystem. PLoS ONE, 8, e65435.

Smith, C.R. \& Baco, A.R. (2003). Ecology of whale falls at the deep-sea floor. Oceanography and Marine Biology: an Annual Review, 311-354.

Sogard, S.M. \& Olla, B.L. (1996). Diel patterns of behavior in juvenile walleye pollock, Theragra chalcogramma. Environmental Biology of Fishes, 47, 379-386.

Steidinger, K.A. \& Tangen, K. (1996). Chapter 3 - Dinoflagellates. In: Identifying Marine Diatoms and Dinoflagellates (ed. Tomas, C.R.). Academic Press, San Diego, pp. 387-584.

Stephenson, B. \& Boertje, R. (2008). Wolf Species Profile. Wildlife Notebook Series. Alaska Department of Fish and Game.

Stewart, J., Ballinger, Giles \& Ferrell, Doug. (2010). Review of the biology and fishery for Australian sardines (Sardinops sagax) in New South Wales ( No. 26). Fisheries Research Report Series. Industry and Investment NSW.

Stocker, R., Seymour, J.R., Samadani, A., Hunt, D.E. \& Polz, M.F. (2008). Rapid chemotactic response enables marine bacteria to exploit ephemeral microscale nutrient patches.

Proceedings of the National Academy of Sciences, 105, 4209-4214.

Sutherland, K.R., Costello, J.H., Colin, S.P. \& Dabiri, J.O. (2014). Ambient fluid motions influence swimming and feeding by the ctenophore Mnemiopsis leidyi. Journal of Plankton Research, 36, 1310-1322.

Taylor, B.L., Chivers, S.J., Larese, J. \& Perrin, W.F. (2007). Generation length and percent mature estimates for IUCN assessments of cetaceans (Administrative Report No. LJ-07-01). National Marine Fisheries Service, Southwest Fisheries Science Center.

Thompson, A.M., Durbin, E.G. \& Durbin, A.G. (1994). Seasonal changes in maximum ingestion rate of Acartia tonsa in Narragansett Bay, Rhode Island, USA. Marine Ecology Progress Series, 108, 91-105.

Tirmenstein, D.A. (1991). Quercus rubra. Available at: https://www.fs.fed.us/database/feis/plants/tree/querub/all.html. Last accessed 28 March 2019. 
Tiselius, P. (1992). Behavior of Acartia tonsa in patchy food environments. Limnology and Oceanography, 37, 1640-1651.

Toft, J.D., Cordell, J.R., Simenstad, C.A. \& Stamatiou, L.A. (2007). Fish distribution, abundance, and behavior along city shoreline types in Puget Sound. North American Journal of Fisheries Management, 27, 465-480.

Trites, A.W. \& Bigg, M.A. (1996). Physical growth of northern fur seals (Callorhinus ursinus): seasonal fluctuations and migratory influences. Journal of Zoology, 238, 459-482.

Urmy, S. \& Warren, J. (2018). Foraging hotspots of common and roseate terns: the influence of tidal currents, bathymetry, and prey density. Marine Ecology Progress Series, 590, 227-245.

Urmy, S.S. (2021). Visual trail following in colonial seabirds: theory, simulation, and remote observations. Ecological Monographs, 91, 1-20.

Urmy, S.S. \& Warren, J.D. (2017). Quantitative ornithology with a commercial marine radar: standard-target calibration, target detection and tracking, and measurement of echoes from individuals and flocks. Methods in Ecology and Evolution, 8, 860-869.

Urmy, S.S. \& Warren, J.D. (2019). Seasonal changes in the biomass, distribution, and patchiness of zooplankton and fish in four lakes in the Sierra Nevada, California. Freshwater Biology, 64, 1692-1709.

Urmy, S.S. \& Warren, J.D. (2020). Evaluating the target-tracking performance of scanning avian radars by augmenting data with simulated echoes. Methods in Ecology and Evolution, 11, 559-569.

U.S. Fish and Wildlife Service. (1997). Southeastern Beach Mouse Perimyscus polio niveiventris. Multi-species recovery plan for South Florida. U.S. Fish and Wildlife Service.

Van Voorhies, W.A., Khazaeli, A.A. \& Curtsinger, J.W. (2004). Lack of correlation between body mass and metabolic rate in Drosophila melanogaster. Journal of Insect Physiology, 50, 445-453.

Vaughn, C.R. (1985). Birds and insects as radar targets: A review. Proceedings of the IEEE, 73, 205-227.

Verburg, P. \& Albert, A. (2018). Lake Taupo long term monitoring programme - 2016-2017 (Technical Report No. 12711725). Waikato Regional Council, Hamilton, NZ.

Vrijenhoek, R.C., Johnson, S.B. \& Rouse, G.W. (2008). Bone-eating Osedax females and their 'harems' of dwarf males are recruited from a common larval pool. Molecular Ecology, 17, 4535-4544. 
Waggett, R.J. \& Buskey, E.J. (2006). Copepod sensitivity to flow fields: detection by copepods of predatory ctenophores. Marine Ecology Progress Series, 323, 205-211.

Weston, D.E. \& Andrews, H.W. (1990). Seasonal sonar observations of the diurnal shoaling times of fish. The Journal of the Acoustical Society of America, 87, 673-680.

Whitton, T.A., Jenkins, S.R., Richardson, C.A. \& Hiddink, J.G. (2012). Aggregated prey and predation rates: Juvenile shore crabs (Carcinus maenas) foraging on post-larval cockles (Cerastoderma edule). Journal of Experimental Marine Biology and Ecology, 432-433, 29-36.

Wilkinson, E.B., Branch, L.C. \& Miller, D.L. (2013). Functional habitat connectivity for beach mice depends on perceived predation risk. Landscape Ecology, 28, 547-558.

Williams, R. \& Noren, D.P. (2009). Swimming speed, respiration rate, and estimated cost of transport in adult killer whales. Marine Mammal Science, 25, 327-350.

Williams, R., Trites, A.W. \& Bain, D.E. (2002). Behavioural responses of killer whales (Orcinus orca) to whale-watching boats: opportunistic observations and experimental approaches. Journal of Zoology, 256, 255-270.

Williams, T.M. \& Heard, D.C. (1986). World status of wild Rangifer tarandus populations. Rangifer, 19-28.

Woodby, D.A. (1984). The April distribution of murres and prey patches in the southeastern Bering Sea: Murres and prey patches. Limnology and Oceanography, 29, 181-188.

Wright, S., Thomas, D., Marchant, H., Higgins, H., Mackey, M. \& Mackey, D. (1996). Analysis of phytoplankton of the Australian sector of the Southern Ocean: comparisons of microscopy and size frequency data with interpretations of pigment HPLC data using the "CHEMTAX" matrix factorization program. Marine Ecology Progress Series, 144, 285-298.

Yawata, Y., Carrara, F., Menolascina, F. \& Stocker, R. (2020). Constrained optimal foraging by marine bacterioplankton on particulate organic matter. PNAS, 117, 25571-25579.

Zhang, J., O’Reilly, K.M., Perry, G.L.W., Taylor, G.A. \& Dennis, T.E. (2015). Extending the Functionality of Behavioural Change-Point Analysis with k-Means Clustering: A Case Study with the Little Penguin (Eudyptula minor). PLOS ONE, 10, e0122811. 